\title{
BRD2 inhibition blocks SARS-CoV-2 infection by reducing transcription of the host cell receptor ACE2
}

\author{
Avi J. Samelson ${ }^{1,2}$, Quang Dinh Tran ${ }^{3,4}$, Rémy Robinot ${ }^{5}$, Lucia Carrau ${ }^{6}$, Veronica V. Rezelj ${ }^{3}$, \\ Alice Mac Kain ${ }^{3,4}$, Merissa Chen ${ }^{1,2}$, Gokul N. Ramadoss ${ }^{7,8}$, Xiaoyan Guo ${ }^{1,2}$, Shion A. Lim ${ }^{9,19}$, \\ Irene Lui ${ }^{9}{ }^{9}$, James K. Nuñez ${ }^{10,11}$, Sarah J. Rockwood (1)7, Jianhui Wang ${ }^{12}$, Na Liu' ${ }^{12}$, \\ Jared Carlson-Stevermer ${ }^{13}$, Jennifer Oki ${ }^{13}$, Travis Maures ${ }^{13}$, Kevin Holden ${ }^{13}{ }^{13}$, \\ Jonathan S. Weissman (10,11,14,15, James A. Wells ${ }^{20,9,10}$, Bruce R. Conklin 7,10,16,17, Benjamin R. TenOever (i)6, \\ Lisa A. Chakrabarti ${ }^{5}$, Marco Vignuzzi ${ }^{3}{ }^{3}$, Ruilin Tian ${ }^{1,2,12} \bowtie$ and Martin Kampmann $\mathbb{1}^{1,2,18 \bowtie}$
}

\begin{abstract}
SARS-CoV-2 infection of human cells is initiated by the binding of the viral Spike protein to its cell-surface receptor ACE2. We conducted a targeted CRISPRi screen to uncover druggable pathways controlling Spike protein binding to human cells. Here we show that the protein BRD2 is required for ACE2 transcription in human lung epithelial cells and cardiomyocytes, and BRD2 inhibitors currently evaluated in clinical trials potently block endogenous ACE2 expression and SARS-CoV-2 infection of human cells, including those of human nasal epithelia. Moreover, pharmacological BRD2 inhibition with the drug ABBV-744 inhibited SARS-CoV-2 replication in Syrian hamsters. We also found that BRD2 controls transcription of several other genes induced upon SARS-CoV-2 infection, including the interferon response, which in turn regulates the antiviral response. Together, our results pinpoint BRD2 as a potent and essential regulator of the host response to SARS-CoV-2 infection and highlight the potential of BRD2 as a therapeutic target for COVID-19.
\end{abstract}

T he ongoing COVID-19 pandemic is a public health emergency. As of September 2021, SARS-CoV-2, the novel coronavirus causing this disease, has infected over 200 million people worldwide, causing at least four and a half million deaths (https:// covid19. who.int). New infections are still rapidly increasing, despite current vaccination programmes. The emergence of novel viral variants with the potential to partially overcome vaccine-elicited immunity highlights the need to elucidate the molecular mechanisms that underlie SARS-CoV-2 interactions with host cells to enable the development of therapeutics to treat and prevent COVID-19, complementing ongoing vaccination efforts.

SARS-CoV-2 entry into human cells is initiated by the interaction of the viral Spike protein with its receptor on the cell surface, angiotensin-converting enzyme 2 (ACE2). To uncover new therapeutic targets targeting this step of SARS-CoV-2 infection, we have conducted a focused CRISPR interference (CRISPRi)-based screen for modifiers of Spike binding to human cells. We expected that ACE2 and factors regulating ACE2 expression would be major hit genes in this screen. A second motivation for identifying regulators of ACE2 was the fact that ACE2 affects inflammatory responses and is itself regulated in the context of inflammation ${ }^{1-3}$. Inflammatory signalling, in particular the type I interferon response, is known to be misregulated in the most severely affected patients with COVID-19 $9^{4-7}$. Therefore, regulators of ACE2 expression would probably be relevant for COVID-19 in human patients, as suggested by clinical data ${ }^{8}$.

Previous CRISPR screens have been performed in cell-based models of SARS-CoV-2 infection that overexpressed an ACE2 transgene ${ }^{9,10}$, represented cell types not primarily targeted by SARS-CoV- $2^{11}$ or were non-human cells ${ }^{12}$. Although these studies elucidated major features of SARS-CoV-2 biology, we reasoned that the cell lines used would not have enabled the discovery of regulators of ACE2 expression in relevant human cell types.

We selected a lung epithelial cancer cell line, Calu-3, which endogenously expresses ACE2, to perform a targeted CRISPRi screen to find regulators of Spike protein binding. We found that the strongest hit genes are potent regulators of ACE2 levels. Knockdown

${ }^{1}$ Institute for Neurodegenerative Diseases, University of California San Francisco, San Francisco, CA, USA. ${ }^{2}$ Chan-Zuckerberg Biohub, San Francisco, CA, USA. ${ }^{2}$ Viral Populations and Pathogenesis Unit, Institut Pasteur, Paris, France. ${ }^{4}$ École Doctorale BioSPC, Université de Paris, Sorbonne Paris Cité, Paris, France. ${ }^{5}$ Institut Pasteur, CIVIC Group, Virus and Immunity Unit, Université de Paris, Paris, France. ${ }^{6}$ Microbiology Department, NYU-Langone, New York, NY, USA. ${ }^{7}$ Gladstone Institutes, San Francisco, CA, USA. ${ }^{8}$ Biomedical Sciences PhD Program, University of California San Francisco, San Francisco, CA, USA. ${ }^{9}$ Department of Pharmaceutical Chemistry, University of California San Francisco, San Francisco, CA, USA. ${ }^{10}$ Department of Cellular and Molecular Pharmacology, University of California San Francisco, San Francisco, CA, USA. "Howard Hughes Medical Institute, University of California San Francisco, San Francisco, CA, USA. ${ }^{12}$ School of Medicine, Southern University of Science and Technology, Shenzhen, China. ${ }^{13}$ Synthego Corporation, Redwood City, CA, USA. ${ }^{14}$ Whitehead Institute for Biomedical Research, Cambridge, MA, USA. ${ }^{15}$ Department of Biology, Massachusetts Institute of Technology, Cambridge, USA. ${ }^{16}$ Department of Ophthalmology, University of California San Francisco, San Francisco, CA, USA. ${ }^{17}$ Department of Medicine, University of California San Francisco, San Francisco, CA, USA. ${ }^{18}$ Department of Biochemistry and Biophysics, University of California San Francisco, San Francisco, CA, USA. ${ }^{19}$ Present address: Department of Antibody Engineering, Genentech Inc., San Francisco, CA, USA. $\otimes_{e}$-mail: tianrl@sustech.edu.cn; 

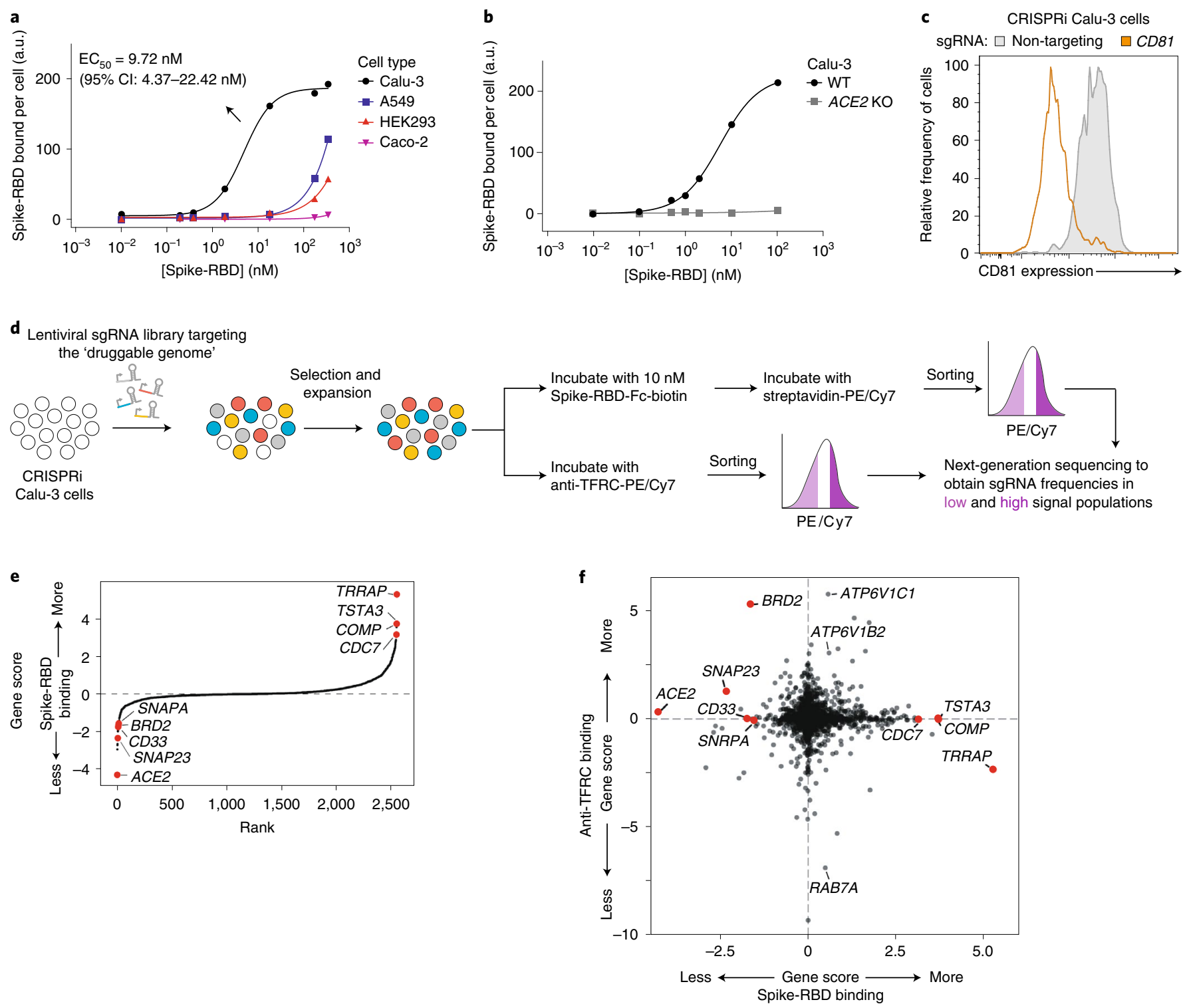

Fig. 1 | CRISPRi screen reveals cellular factors controlling Spike protein binding. a, Human cell lines were incubated with different concentrations of recombinant SARS-CoV-2 Spike-RBD, and the amount of Spike-RBD bound per cell was quantified by flow cytometry. Only Calu-3 cells (black) display saturable binding, for which an $\mathrm{EC}_{50}$ value was fit. The error of $\mathrm{EC}_{50}$ is the $95 \%$ confidence interval. $\mathbf{b}$, Spike-RBD binding is eliminated in Calu-3 cells with ACE2 knockout (KO, grey). c, Validation of CRISPRi activity of a Calu-3 CRISPRi line. Calu-3 cells stably expressing CRISPRi machinery were transduced with an sgRNA targeting CD81 (orange) or a non-targeting sgRNA (grey), and CD81 levels were determined by flow cytometry. The experiments in a-c were performed once. d, CRISPRi screen strategy. CRISPRi Calu-3 cells transduced with an sgRNA library targeting the 'druggable genome' were stained either with Spike-RBD or an anti-TFRC antibody. Cells were then FACS-sorted into bins (top and bottom 30\%) based on Spike-RBD or anti-TFRC binding signal, and frequencies of cells expressing each sgRNA were determined for each bin by targeted next-generation sequencing. The CRISPRi screen was performed once. e, Rank-order plot of Spike-RBD hit genes. Genes selected for follow-up experiments are highlighted as red dots. $\mathbf{f}$, Scatter plot of gene scores for the Spike-RBD screen ( $x$ axis) and the anti-TFRC screen ( $y$ axis). Genes selected for follow-up experiments are highlighted as red dots.

of these genes reduced or increased ACE2 levels transcriptionally, and prevented or enhanced, respectively, SARS-CoV-2 infection in cell culture.

We identified the transcriptional regulator bromodomaincontaining protein 2 (BRD2) as a major node for host-SARS-CoV-2 interaction. BRD2 is part of the bromodomain and extra-terminal domain (BET) family of proteins that includes Brd3, Brd4 and BrdT. BETs are being explored as targets for a number of cancers ${ }^{13}$. These proteins are known to be master transcriptional regulators and serve to bridge chromatin marks (mostly acetyl-lysines) to the transcriptional machinery ${ }^{14}$. We found BRD2 inhibition to downregulate ACE2 expression in Calu-3 cells, induced pluripotent stem cell (iPSC)-derived cardiomyocytes, primary human lung epithelial cells and reconstructed human nasal epithelia. Inhibition of BRD2 with small molecules, some of which are in phase I clinical trials, inhibited SARS-CoV-2 infection in primary human nasal epithelia and in Syrian hamsters. We propose BRD2 as a key regulator and potential therapeutic target for COVID-19.

\section{Results}

CRISPRi screen for Spike-RBD binding to cells. To identify cellular mechanisms controlling the binding of SARS-CoV-2 to human 
a

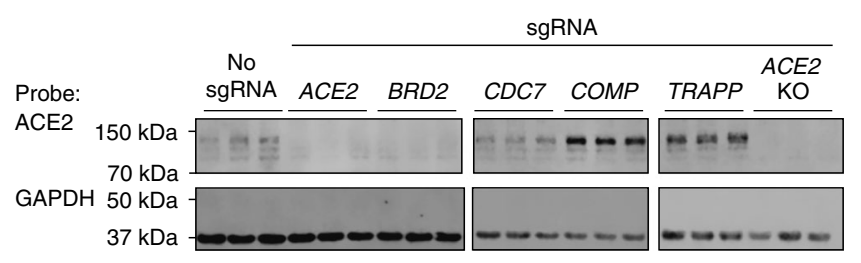

b

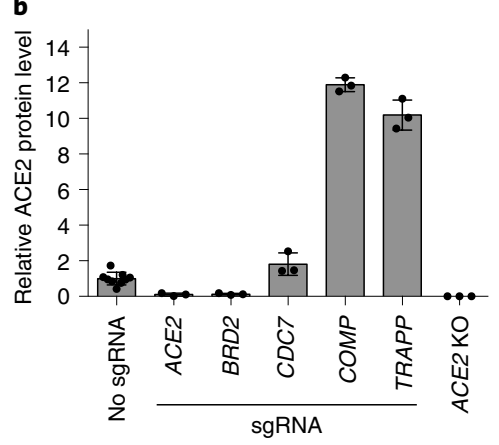

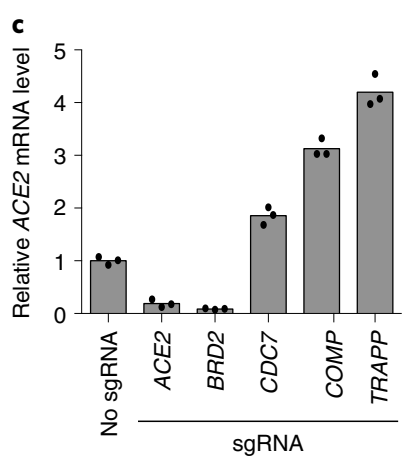

d

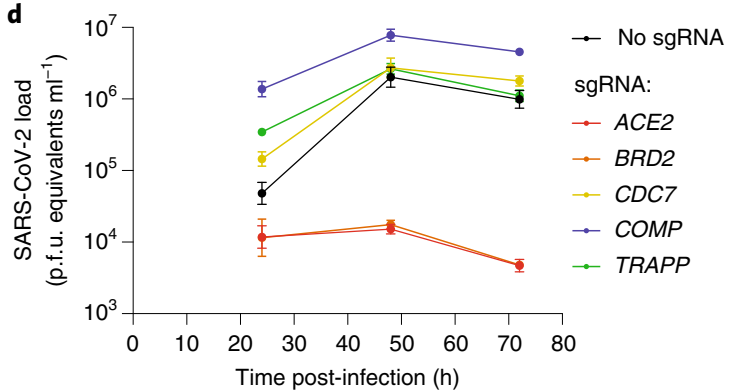

e
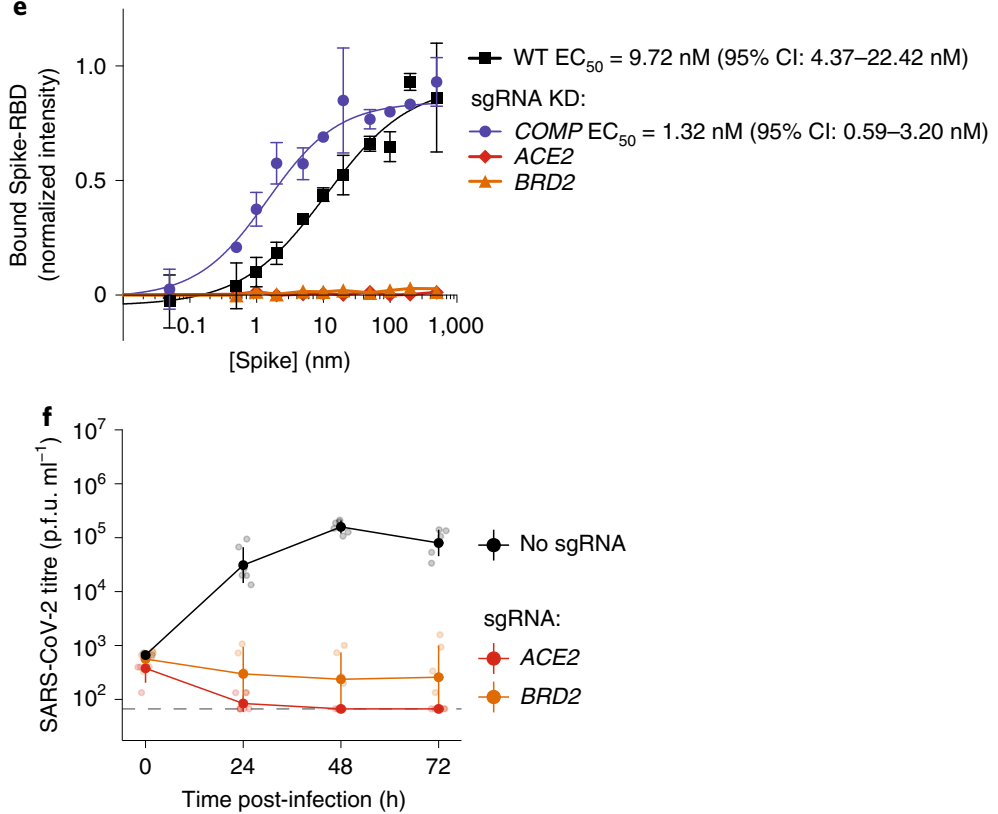

Fig. 2 | Hit genes modulate ACE2 levels and SARS-CoV-2 infection. a, Western blotting for ACE2 and GAPDH in Calu-3 CRISPRi cells expressing no sgRNA or sgRNAs targeting different hit genes, or ACE2 knockout Calu-3 cells. The three lanes represent biological triplicates for each cell line. b, Quantification of ACE2 protein levels relative to GAPDH based on the data in a. Average normalized ACE2 intensity as a fraction of no sgRNA and the standard deviation for three biological replicates are shown. c, Relative amounts of ACE2 transcript levels measured by qPCR in Calu-3 CRISPRi cells expressing sgRNAs targeting different hit genes, compared to cells without sgRNA. Average relative gene expression of three technical replicates is shown from a single experiment. d, Calu-3 CRISPRi cells expressing different sgRNAs targeting hit genes were infected with SARS-CoV-2 and viral RNA in the supernatant was measured by RT-qPCR as a function of time post-infection. The average SARS-CoV-2 load and the standard deviation of three biological replicates are shown. e, Spike-RBD binding to Calu-3 cells was quantified by flow cytometry of Calu-3 cells expressing sgRNAs targeting individual hit genes after incubation with increasing concentrations of Spike-RBD. For genes for which data could be fitted with a binding curve, the $E C_{50}$ was determined along with the $95 \%$ confidence intervals. Data points are average values from three biological replicates for each gene knockdown, with error bars representing the standard deviation, except for ACE2 and BRD2, where only one experiment at each concentration was performed. f, Plaque assays in Calu-3 CRISPRi cells expressing different sgRNAs targeting hit genes were infected with SARS-CoV-2 as a function of time post-infection. Average SARS-CoV-2 titre and the standard deviation of six biological replicates are shown.

cells, we identified a cell line that would robustly bind the viral Spike protein (S). We measured binding of a previously described recombinant protein construct encompassing the SARS-CoV-2 Spike protein receptor-binding domain (RBD) with a C-terminal human immunoglobulin-G (IgG) Fc-domain fusion ${ }^{15}$, referred to hereafter as Spike-RBD, to several commonly used human cell lines (Fig. 1a and Extended Data Fig. 1a). Within this cell line panel, only Calu-3 cells displayed a binding curve consistent with specific binding of Spike-RBD, with a half-maximum effective concentration $\left(\mathrm{EC}_{50}\right)$ of $9.72 \mathrm{nM}$ (95\% CI: 4.37-22.42 nM). This value agrees with the dissociation constant of Spike-RBD-ACE2 binding determined in vitro $(4.7 \mathrm{nM})^{16}$, within measurement error. Spike-RBD binding is dependent on ACE2 expression, as binding is abrogated in Calu-3 ACE2 knockout cells (Fig. 1b). Calu-3 cells are challenging to culture compared to most cell lines - they proliferate slowly, and their adherence properties pose challenges for flow cytometry. Nevertheless, Calu-3 cells are a particularly attractive cell culture model for studying SARS-CoV-2 from the biological point of view, because they are derived from lung epithelia, which is selectively infected by SARS-CoV-2 in patients ${ }^{17}$, are known to support infection of SARS-CoV and SARS-CoV- $2^{18}$ and have recently been reported to closely recapitulate gene-expression changes that occur in patients ${ }^{19}$.

We generated a polyclonal Calu-3 line constitutively expressing machinery to enable CRISPRi-based genetic screens ${ }^{20,21}$ and validated its CRISPRi activity (Fig. 1c and Extended Data Fig. 1b). CRISPRi uses a catalytically dead Cas9 (dCas9) fused to a transcriptional repressor, KRAB, to knock down genes at specific sites programmed by the loading of the dCas9-KRAB with a single-guide 
RNA (sgRNA). Using this line, we then performed a focused CRISPRi screen for factors controlling Spike-RBD binding (Fig. 1d). To maximize our chances of identifying potential undiscovered therapeutic targets for COVID-19, we screened a sgRNA library targeting the 'druggable genome', comprising 2,300 genes, with $\sim 16,000$ total sgRNAs including non-targeting control sgRNAs ${ }^{22}$. In parallel, we screened the same library using a fluorophore-conjugated antibody against the transferrin receptor (TFRC, also known as CD71) to control for factors that generally affect protein trafficking or protein binding to the cell surface (Fig. 1d-f). Owing to the limitations of the Spike-RBD binding assay and Calu-3 cells, this screen was conducted at a lower average representation $(\sim 100$ sorted cells per sgRNA) than ideal, resulting in relatively high noise and therefore fewer hits crossing a false discovery rate cutoff of 0.1 than in typical CRISPR screens (Extended Data Fig. 1c and Methods).

Despite the increased noise, $A C E 2$, as expected, was the strongest hit gene, knockdown of which decreased binding of SpikeRBD, while having no effect on TFRC levels (Fig. 1e,f). Conversely, $R A B 7 A$, which was recently reported to be essential for the trafficking of TFRC to the cell surface ${ }^{23}$, was the strongest hit that decreased TFRC levels, with no effect on Spike-RBD binding (Fig. 1f). Generally, hits were not correlated between the two screens (Fig. 1f), demonstrating the specificity of each screen. Although the screens did not result in a large number of strong hits (Supplementary Table 1), we decided to validate the top 15 genes for which knockdown decreased Spike-RBD binding and the top five genes for which knockdown increased Spike-RBD binding. We cloned individual sgRNAs targeting each of these genes and evaluated their effect on Spike-RBD binding (Extended Data Fig. 2). Based on these experiments, we selected hits that robustly recapitulated their phenotypes from the primary screen for further characterization: two genes for which knockdown decreased Spike-RBD binding (ACE2 and BRD2) and three genes for which knockdown increased Spike-RBD-binding (CDC7, COMP and TRRAP).

Hit genes modulate ACE2 levels and infection with SARS-CoV-2. Because Spike-RBD binding is dependent on ACE2 expression, we hypothesized that other hit genes might act by modulating ACE2 levels. Western blots for ACE2 levels in Calu-3 cell lines expressing sgRNAs against validated target genes (hereafter referred to as knockdown lines) indeed revealed marked changes in ACE2 protein levels. For hits associated with lower levels of Spike-RBD binding in the primary screen, we observed lower levels of ACE2 protein, and vice versa for those hits associated with higher levels of Spike-RBD binding (Fig. 2a,b). To distinguish whether hit genes affected ACE2 protein levels via transcriptional or post-transcriptional mechanisms, we performed quantitative polymerase chain reaction (qPCR) to measure ACE2 transcript levels in these same knockdown lines. For all tested genes, we observed changes in $A C E 2$ transcript levels that were concordant with the changes in ACE2 protein levels (Fig. 2c), indicating that they acted at the transcriptional level. Some genes, such as COMP and TRAPP, showed relatively modest effects on ACE2 transcript levels, but quite large effects on ACE2 protein levels, suggesting that these hit genes additionally affect post-transcriptional regulation of ACE2 expression.

We next determined the effect of hit gene knockdown on susceptibility to SARS-CoV-2 infection. We infected cells expressing sgRNAs against hit genes with SARS-CoV-2 and measured virus replication 24,48 and $72 \mathrm{~h}$ post-infection using qPCR with reverse transcription (RT-qPCR; Fig. 2d). Already at $24 \mathrm{~h}$ post-infection, viral genome copies diverged concordantly with changes in ACE2 levels and Spike-RBD binding: sgRNAs that lowered Spike-RBD binding reduced virus replication, and sgRNAs that increased Spike-RBD binding resulted in higher virus replication. BRD2 knockdown abrogated viral replication in these cells to levels similar to ACE2 knockdown, even at $72 \mathrm{~h}$ post-infection, and COMP knockdown supported an order of magnitude increase in viral titres. Focusing on these three hit genes-ACE2, BRD2 and COMP-we then quantified how gene knockdown modulates Spike-RBD binding to cells (Fig. 2e). Knockdown of ACE2 and BRD2 abolished spike binding, while COMP decreased the $\mathrm{EC}_{50}$ compared to wild type (WT) from $9.72 \mathrm{nM}$ (95\% CI: $4.37-22.42 \mathrm{nM})$ to $1.32 \mathrm{nM}(95 \%$ CI: $0.59-3.20 \mathrm{nM}$ ), by almost a full order of magnitude (Fig. 2e). We also confirmed that gene knockdown decreased SARS-CoV-2 replication by performing plaque assays on WT, BRD2 KD and ACE2 knockdown cells (Fig. 2f).

BRD2 inhibitors prevent SARS-CoV-2 infection of human cells. Given the stringent inhibition of SARS-CoV-2 infection achieved by $B R D 2$ knockdown, and the fact that BRD2 is currently being evaluated as a therapeutic target in cancer ${ }^{13,24}$, with several small-molecule inhibitors in clinical trials ${ }^{25}$, we decided to focus on this hit gene.

We validated that CRISPRi knockdown of BRD2 robustly reduced BRD2 protein levels (Extended Data Fig. 3). Transgenic expression of full-length $\mathrm{BRD} 2$ restored ACE2 transcript levels (Fig. 3a), validating that the reduction in ACE2 expression triggered by CRISPRi targeting of $B R D 2$ was indeed due to $B R D 2$ knockdown. Transgenic expression of truncation mutants of $B R D 2$ did not rescue ACE2 expression (Fig. 3a), indicating that full-length BRD2 is required for ACE2 expression.

To test the potential of BRD2 as a therapeutic target for COVID19 , we treated cells with a panel of compounds targeting BRD2: two $\mathrm{BET}$-domain inhibitors (JQ1 ${ }^{26}$ and $\mathrm{ABBV}-744^{27}$; the latter is currently in clinical trials NCT03360006 and NCT04454658) and three proteolysis targeting chimaeric (PROTAC) compounds that lead to the degradation of BRD2 (dBET- $6^{28}$, ARV-771 ${ }^{29}$ and BETd-260 29 ).

Fig. 3 | BRD2 inhibitors potently reduce ACE2 levels and SARS-CoV-2 infection. a, Transgenic constructs expressed in Calu-3 cells (left), and transcript levels of $A C E 2$ relative to $A C T B$ in Calu-3 cells transduced with enhanced green fluorescent protein-BRD2 truncations in a BRD2 knockdown background (right). Average relative ACE2 gene expression compared to non-transduced cells and the standard deviation of $n=3$ biological replicates are shown. aa, amino acid. b. Transcript levels of ACE2 relative to ACTB in Calu-3 cells treated with BRD2 inhibitors (JQ1 at $10 \mu \mathrm{M}, \mathrm{ABBV}-744$ at $10 \mu \mathrm{M}$ and dBET-6 at $200 \mathrm{nM}$ ) were quantified at $24 \mathrm{~h}$ (dark grey bars) and $72 \mathrm{~h}$ (light grey bars) post-treatment. Average ACE2 mRNA levels relative to vehicle-treated of three technical replicates are shown from a single experiment. c, Transcript levels of ACE2 relative to ACTB in primary human bronchial epithelial cells treated (NHBE) with BRD2 inhibitors (JQ1 at $10 \mu \mathrm{M}, \mathrm{dBET}-6$ at $20 \mathrm{nM}, \mathrm{ABBV}-744$ at 0.01-10 $\mu \mathrm{M}$ ) were quantified at $72 \mathrm{~h}$ post-treatment. Average ACE2 mRNA levels relative to vehicle and the standard deviation of $n=3$ biological replicates are shown, except for vehicle-treated, for which $n=6$ replicates are shown. d, Transcript levels of ACE2 relative to 18S ribosomal RNA (rRNA) in human iPSC-derived cardiomyocytes treated with the indicated concentrations of BRD2 inhibitors were quantified at $72 \mathrm{~h}$ post-treatment. Average ACE2 mRNA levels relative to vehicle-treated and the standard deviation of $n=3$ biological replicates for each condition are shown, except for the vehicle-treated sample, for which $n=6$ biological replicates are shown. e, SARS-CoV-2 viral RNA in supernatant measured by RT-qPCR at $24 \mathrm{~h}$ post-infection of Calu- 3 cells. Infection took place $72 \mathrm{~h}$ after treatment with the indicated concentrations of BRD2 inhibitors. Measurements are the average of $n=5$ biological replicates. Error bars show the standard deviation. $\mathbf{f}$, Plaque assays in Calu-3 CRISPRi cells treated with increasing concentrations of the BET inhibitors JQ1 or ABBV-744 infected with SARS-CoV-2 as a function of time post-infection. Average viral titre and the standard deviation of six biological replicates are shown, except for vehicle and untreated, for which three biological replicates are shown. 
After only $24 \mathrm{~h}$ of treatment with these drugs, ACE2 messenger RNA (mRNA) levels measured by qPCR decreased roughly twofold (Fig. 3b). This effect was magnified after treatment for $72 \mathrm{~h}$, when almost no ACE2 mRNA was detectable for any of the BRD2-targeting compounds tested, phenocopying BRD2 knockdown (Fig. 3b). Similarly, we found that BET inhibitors led to substantial decreases in ACE2 mRNA levels in primary human bronchial epithelial cells (Fig. 3c) and human iPSC-derived cardiomyocytes (Fig. 3d) - two non-transformed cell types that are susceptible to SARS-CoV-2 infection $^{30,31}$. Importantly, BET inhibitors were non-toxic to Calu-3 cells, primary human bronchial epithelial cells and cardiomyocytes at effective concentrations (Extended Data Fig. 4).

Because pharmacological inhibition of BRD2 phenocopied BRD2 knockdown, we hypothesized that these same compounds might prevent infection of cells exposed to SARS-CoV-2. To test this, we treated Calu- 3 cells for $72 \mathrm{~h}$ with the BET inhibitors JQ1 and ABBV744 , and measured SARS-CoV-2 replication at $48 \mathrm{~h}$ post-infection. Strikingly, we found that treated cells displayed 100-fold decreased viral replication versus untreated cells (Fig. 3e), a similar effect size to BRD2 or ACE2 knockdown (Fig. 2d,e). a

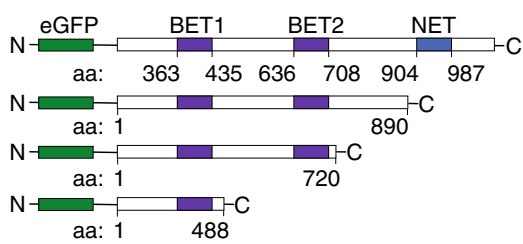

c Primary human
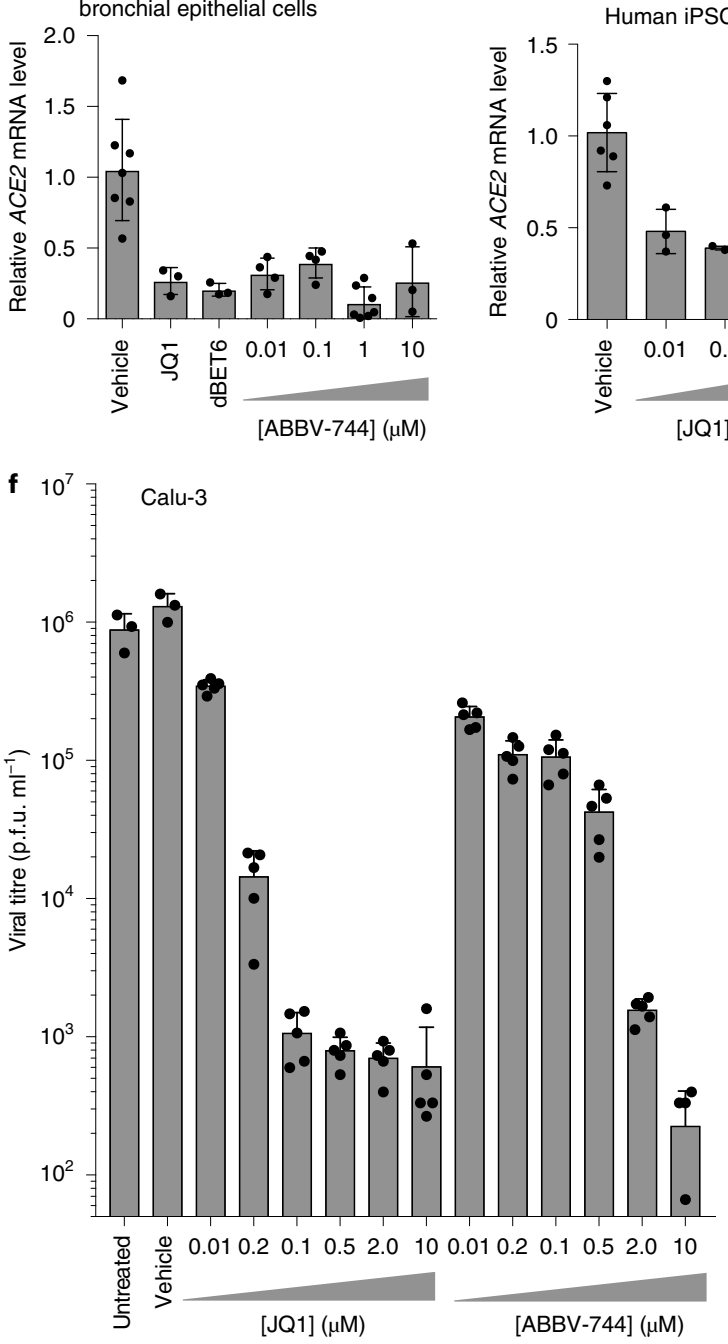
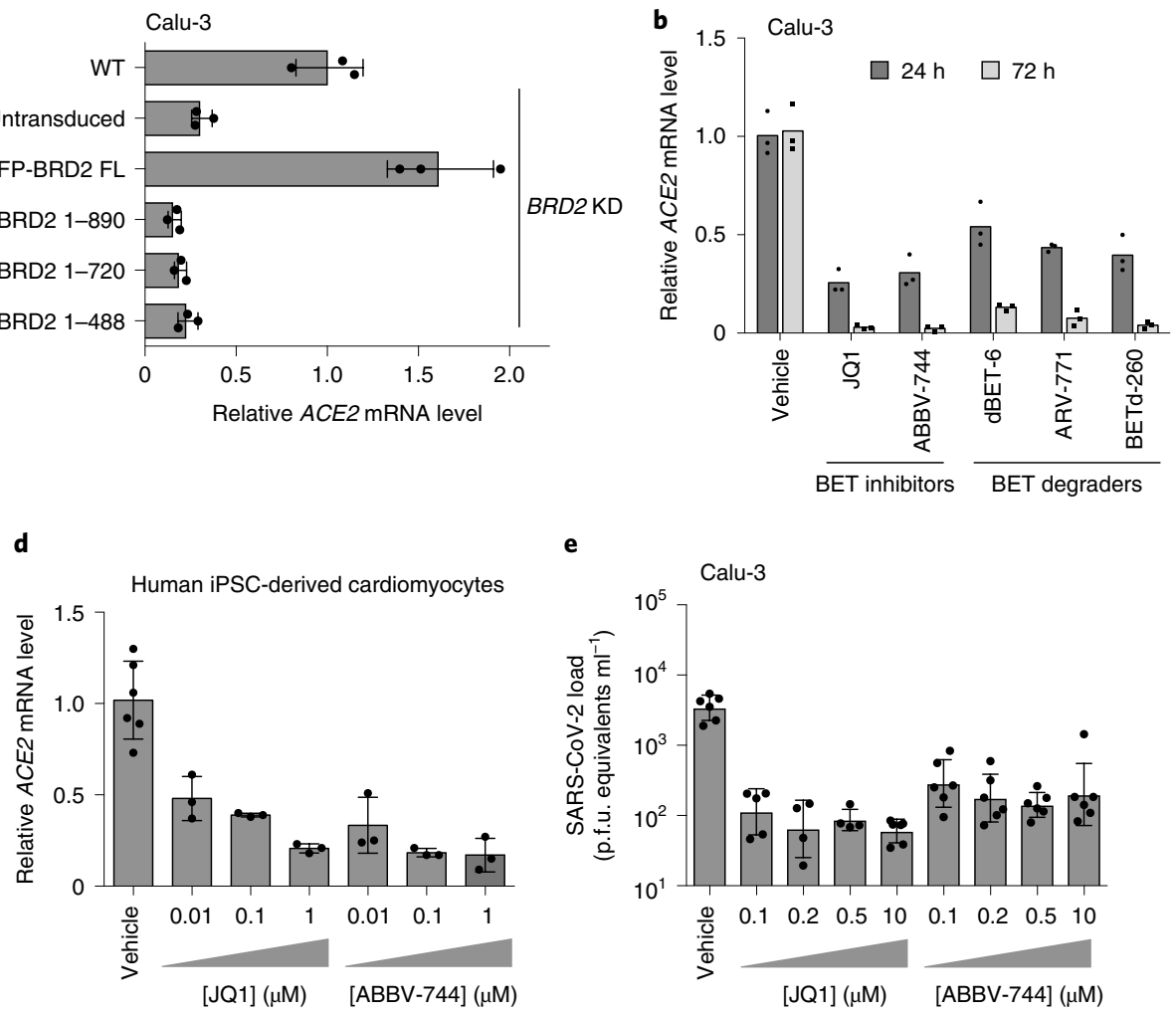
a

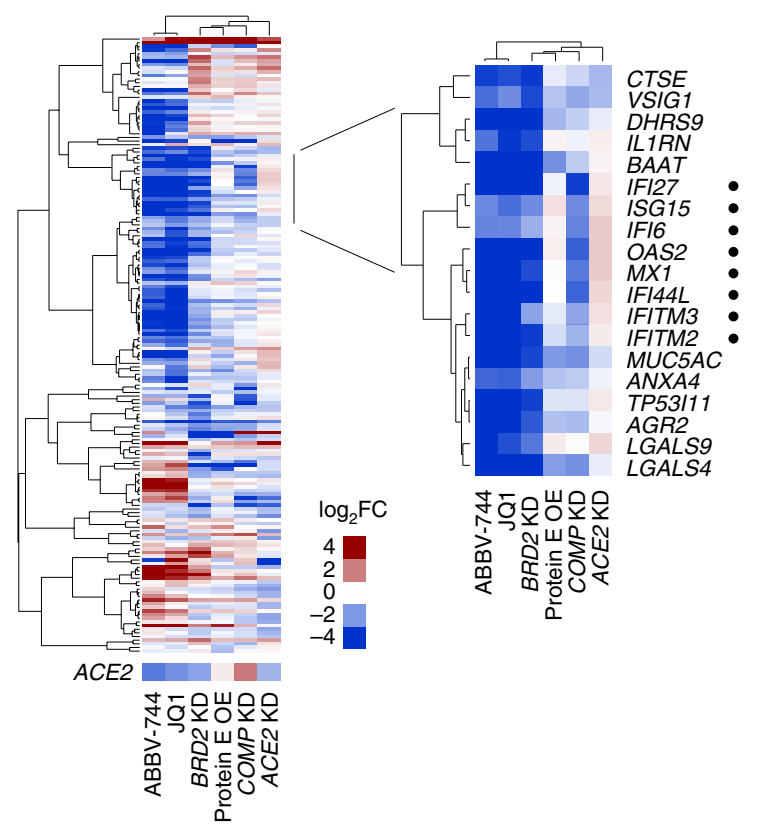

b

GO: biological process

Cellular response to type I interferon (GO:0071357)

Type I interferon signalling pathway (GO:0060337)

Negative regulation of viral genome replication (GO:0045071)

Negative regulation of viral life cycle (GO:1903901)

Regulation of viral genome replication (GO:0045069)

Cytokine-mediated signalling pathway (GO:0019221)

Response to cytokine (GO:0034097)

Regulation of viral entry into host cell (GO:0046596)

Response to type I interferon (GO:0034340)

Response to interferon- $\gamma$ (GO:0034341)

0

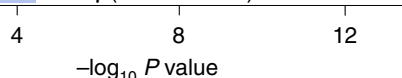

C Downregulated in ABBV-744, JQ1 and

Downregulated in ABBV-744,
BRD2 KD conditions
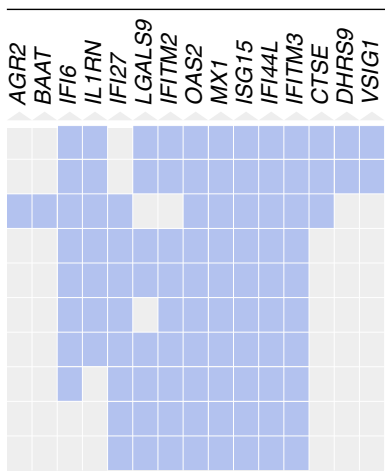

ENRICHR: COVID-19 related gene sets

Healthy lung biopsy vs COVID-19 infected lung series 15 from GSE147507 up genes Upregulated by SARS-CoV-2 in lung tissue from GSE147507 Upregulated by SARS-CoV-2 in intestinal organoids $60 \mathrm{~h}$ expansion from GSE149312 SARS perturbation up genes airway epithelium (HAE) from GSE47961:GPL6480:4 SARS perturbation up genes airway epithelium (HAE) from GSE47961:GPL6480:5 SARS perturbation up genes airway epithelium (HAE) from GSE47961:GPL6480:3 Upregulated by SARS-CoV-2 in NHBE from GSE147507

SARS perturbation up genes airway epithelium (HAE) from GSE47961:GPL6480:6

SARS-CoV perturbation up genes bronchial epithelial 2B4 from GSE17400:GPL570:3 SARS-CoV perturbation up genes bronchial epithelial 2B4 from GSE17400:GPL570:5

\begin{tabular}{c|c|c}
8 & 12 & 10 \\
$-\log _{10} P$ value & &
\end{tabular}
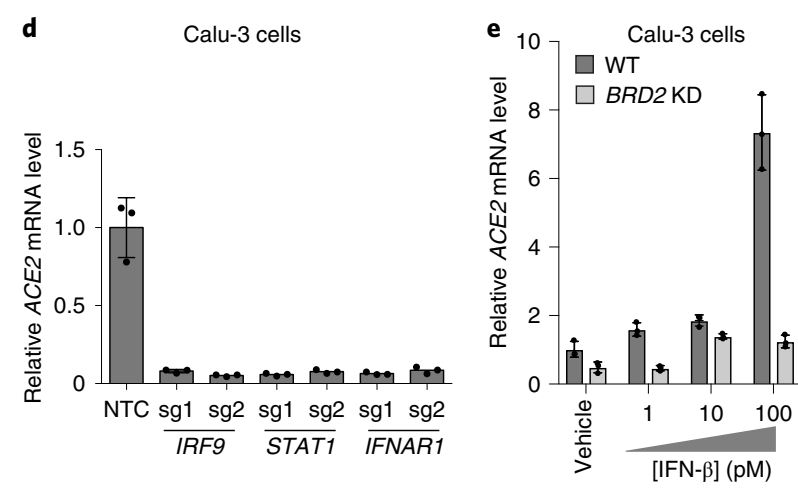

\section{f}

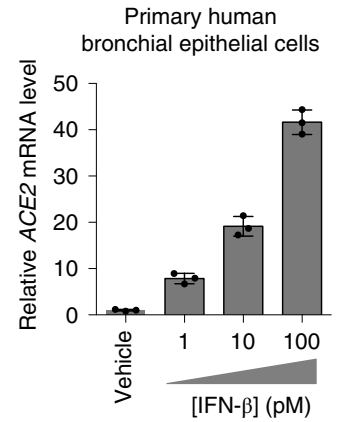

BRD2 regulates ACE2 and SARS-CoV-2-induced host genes. We next asked whether BRD2 controls transcription of additional genes beyond ACE2. We performed RNA-sequencing (RNA-seq) of Calu-3 cells after treatment with the BET-domain inhibitors JQ1 and ABBV-744 as well as BRD2 CRISPRi knockdown (Supplementary Table 2). We also included CRISPRi knockdown of two other validated hit genes from our screen, COMP and ACE2, as well as overexpression of the viral protein $\mathrm{E}$, which has been reported to interact with BRD2 $2^{32}$. RNA-seq of BRD2 knockdown and BET-domain inhibitor treated cells recapitulated downregulation of ACE2 (Fig. 4a). TMPRSS2, the gene encoding a protease important for viral entry in many cell types, was not a differentially expressed gene in any condition (Supplementary Table 2). Surprisingly, BRD2 knockdown or pharmacological inhibition also resulted in marked downregulation of genes involved in the type I interferon response, while $A C E 2$ knockdown slightly increased expression of those same genes 
Fig. 4 | BRD2 controls genes induced by interferon and SARS-CoV-2 infection. a, Differentially expressed genes (DEGs) from RNA-sequencing of Calu-3 cells under different treatment conditions compared to control cells: $72-\mathrm{h}$ treatment with $10 \mu \mathrm{M} \mathrm{JQ1} \mathrm{or} 10 \mu \mathrm{M}$ ABBV-744, BRD2 knockdown (KD), SARS-CoV-2 protein E overexpression (OE), COMP KD and ACE2 KD. The quasi-likelihood method gImQLFTest was implemented in edgeR. $P$ values were adjusted for multiple comparisons using the Benjamini-Hochberg method. The heatmap shows the $\log _{2}$-fold change (log $\mathrm{FC}$ ) relative to untreated controls for each condition (columns) for genes (rows) among the top 50 DEGs (by $P$ values) in at least one condition. ACE2 was not among these genes and is shown as a separate row. Inset: a cluster of genes downregulated upon both BRD2 inhibition and BRD2 knockdown. Among these, genes associated with the Gene Ontology (GO) term 'Cellular Response to Type I interferon' are marked by black circles. b, Significantly enriched (FDR <0.05) GO biological process terms for the genes shown in the inset in a. c. Enrichment analysis for genes in the inset in a reveals COVID-19-related gene sets. Genes associated with a gene set are marked in blue. d, Calu-3 cells expressing sgRNAs knocking down genes essential for interferon signalling assayed for ACE2 gene expression relative to ACTB by qPCR. Average ACE2 mRNA level relative to non-targeting control (NTC) sgRNA and the standard deviation of $n=3$ biological replicates are shown for each condition. e, WT (dark grey) or BRD2 KD (light grey) Calu-3 cells were treated with IFN- $\beta$, and transcript levels of ACE2 relative to $A C T B$ were quantified at $72 \mathrm{~h}$ post-treatment by qPCR. Average ACE2 levels relative to vehicle-treated and the standard deviation of $n=3$ biological replicates are shown for each condition. f, Primary human bronchial epithelial cells (left) and human iPSC-derived cardiomyocytes (right) were treated with the indicated concentrations of IFN- $\beta$, and transcript levels of ACE2 relative to ACTB (for Calu-3 and primary human bronchial epithelial cells) or $18 \mathrm{~S}$ rRNA (cardiomyocytes) were quantified at $72 \mathrm{~h}$ post-treatment by qPCR. Average ACE2 mRNA levels relative to WT and the standard deviation of $n=3$ biological replicates are shown for each condition.

(Fig. 4a,b). Furthermore, the genes downregulated by both BRD2 knockdown and inhibition were strongly enriched in genes induced by SARS-CoV-2 infection in patient and cultured cells (Fig. 4c).

These findings are compatible with two distinct mechanisms: BRD2 could independently regulate ACE2 and SARS-CoV2 -induced interferon response genes, or BRD2 could mediate the response to interferon, which in turn regulates $A C E 2$ transcription. $A C E 2$ expression has been reported to be induced by interferons in some studies ${ }^{1,2}$. Other studies, however, suggest that interferon suppresses ACE2 expression ${ }^{3}$.

In Calu-3 cells, disruption of basal interferon signalling, via knockdown of the genes essential for interferon signal transduction-IRF9, STAT1 or IFNAR1-abrogated ACE2 expression (Fig. 4d and Extended Data Fig. 5). Conversely, treatment with exogenous interferon- $\beta$ (IFN- $\beta$ ) stimulates $A C E 2$ expression in a concentration-dependent manner. Upon BRD2 knockdown, however, this concentration-dependent increase in ACE2 expression is inhibited (Fig. 4e). Thus, BRD2 is required for interferon-induced $A C E 2$ expression. Treatment with IFN- $\beta$ similarly strongly increased ACE2 mRNA levels in primary human bronchial epithelial cells, but reduced ACE2 mRNA levels in human iPSC-derived cardiomyocytes (Fig. 4f), suggesting that the effect of interferons on ACE2 can be context-dependent.

To test whether BRD2 is a direct transcriptional regulator of $A C E 2$, we performed CUT\&RUN ${ }^{33}$ to comprehensively map genomic loci bound by BRD2 in Calu-3 cells (Supplementary Table 3). CUT\&RUN is similar to chromatin immunoprecipitation sequencing (ChIP-seq), as it measures the occupancy of factors bound to DNA, but has the advantage of higher sensitivity and a lower requirement for cell numbers ${ }^{33}$. Genes adjacent to BRD2-bound sites detected in our experiment showed a highly significant overlap with BRD2-bound sites previously mapped by ChIP-seq in NCI-H23 $3^{34}$ cells, another lung epithelium-derived cancer cell line (Fig. 5a). To further validate our CUT\&RUN analysis, we performed binding and expression target analysis ${ }^{35}$ (BETA) to uncover direct BRD2 targets that were differentially expressed upon $B R D 2$ knockdown, and identified several interferon response genes as direct BRD2 targets that were downregulated (Fig. 5b). We verified that a previously described ${ }^{34} \mathrm{BRD} 2$ binding side upstream of PVT1 was also detected in our experiment (Fig. 5c). We also mapped a BRD2 binding site upstream of the several interferon-stimulated genes (ISGs), including IRF9, STAT1 and MX1 (Fig. 5d). Although there was some signal in the WT background at the ACE2 locus that is decreased in BRD2 knockdown cells, there were no peaks as determined by the peak calling algorithm (Fig. 5e), suggesting that $\mathrm{BRD} 2$ is not a direct transcriptional regulator of $A C E 2$ expression.

We also performed CUT\&RUN for histone H2A.Z, which was previously reported to modulate the magnitude of ISG expression and thus connect BRD2 activity to interferon stimulation ${ }^{36}$. We found decreased H2A.Z occupancy at ISGs in BRD2 knockdown cells (Fig. 5 d), recapitulating the role of BRD2 as a potential chaperone of H2A.Z. These results support a model in which BRD2 controls the transcription of key interferon response genes, which can in turn induce ACE2 transcription in some cell types (Fig. 5f). Alternatively, ACE2 expression may be controlled by other genes that are expressed in a BRD2-dependent, interferon-stimulated manner (Fig. 5f).

ABBV-744 reduces infection in primary cells and in vivo. We then tested whether ABBV-744, a bromodomain inhibitor currently in clinical trials, could reduce SARS-CoV-2 infection and infection-associated phenotypes in more physiological models.

First, we investigated a human nasal epithelial model ${ }^{37}$. We treated reconstituted nasal epithelia maintained in air/liquid interphase conditions with $100 \mathrm{nM}$ and $300 \mathrm{nM}$ ABBV-744 (above the reported half-maximum inhibitory concentration $\left(\mathrm{IC}_{50}\right)$ of $4-18 \mathrm{nM}$ )

Fig. 5 | BRD2 directly regulates transcription of interferon-induced genes. a, Genes associated with BRD2 CUT\&RUN peaks within $10 \mathrm{~kb}$ of a transcription start site determined in this study in Calu-3 cells overlap significantly with published BRD2 ChIP-seq peaks from the indicated datasets $(P<0.0001$, two-sided Fisher's exact test). b, BETA was performed to identify direct BRD2 targets that were differentially expressed upon BRD2 knockdown using the one-tailed Kolmogorov-Smirnov test. Many interferon response genes were identified as direct BRD2 targets. Direct BRD2 targets that were downregulated upon BRD2 knockdown were analysed by ENRICHR for enriched Reactome pathways. Pathways with adjusted $P$ values less than 0.05 are displayed. c-e, CUT\&RUN experiments were conducted to map BRD2, H2A.Z and H3K4me3 genomic localization in WT (blue traces) and BRD2 knockdown (red) Calu-3 cells. Each trace represents an independent biological replicate. c, Known BRD2 regulatory sites are recapitulated. Raw signal tracks for WT and BRD2 knockdown cells are shown at the known BRD2 locus PVT1. BRD2 ChIP-seq tracks from human lung cells are shown. BRD2 peaks were called over IgG using SEACR at FDR $<0.05$. d, Identified BRD2 and histone H2A.Z occupancy and peak calling at ISGs in BRD2 knockdown and WT Calu-3 cells. Raw signal tracks for BRD2, histone H2A.Z and H3K4me are shown. BRD2 peaks were called over IgG using SEACR at FDR $<0.05$. BRD2 ChIP-seq tracks from human lung cells are also shown. e, Raw BRD2 signal tracks and peak calling as for $\mathbf{c}$, at the ACE2 locus. f, Proposed model for BRD2 control of ACE2 expression. 
and performed SARS-CoV-2 or mock infections (Fig. 6a). First, we found that ABBV-744 treatment reduced ACE2 levels in these conditions (Fig. 6b). Apical supernatants did not show significant changes in viral RNA concentrations at two or four days post-infection (Extended Data Fig. 6a). Intracellular viral RNA concentrations, however, were significantly decreased in the
ABBV-744 conditions (Fig. 6c). Furthermore, epithelial barrier integrity, as measured by transepithelial electrical resistance (Fig. 6d) and cytotoxicity (Fig. 6e), were rescued in infected cells treated with ABBV-744. Thus, ABBV-744 partially inhibited SARS-CoV-2 replication and fully rescued epithelial barrier integrity in a primary human nasal epithelial model.

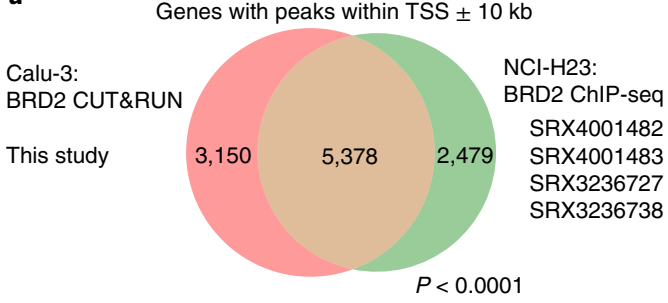

C
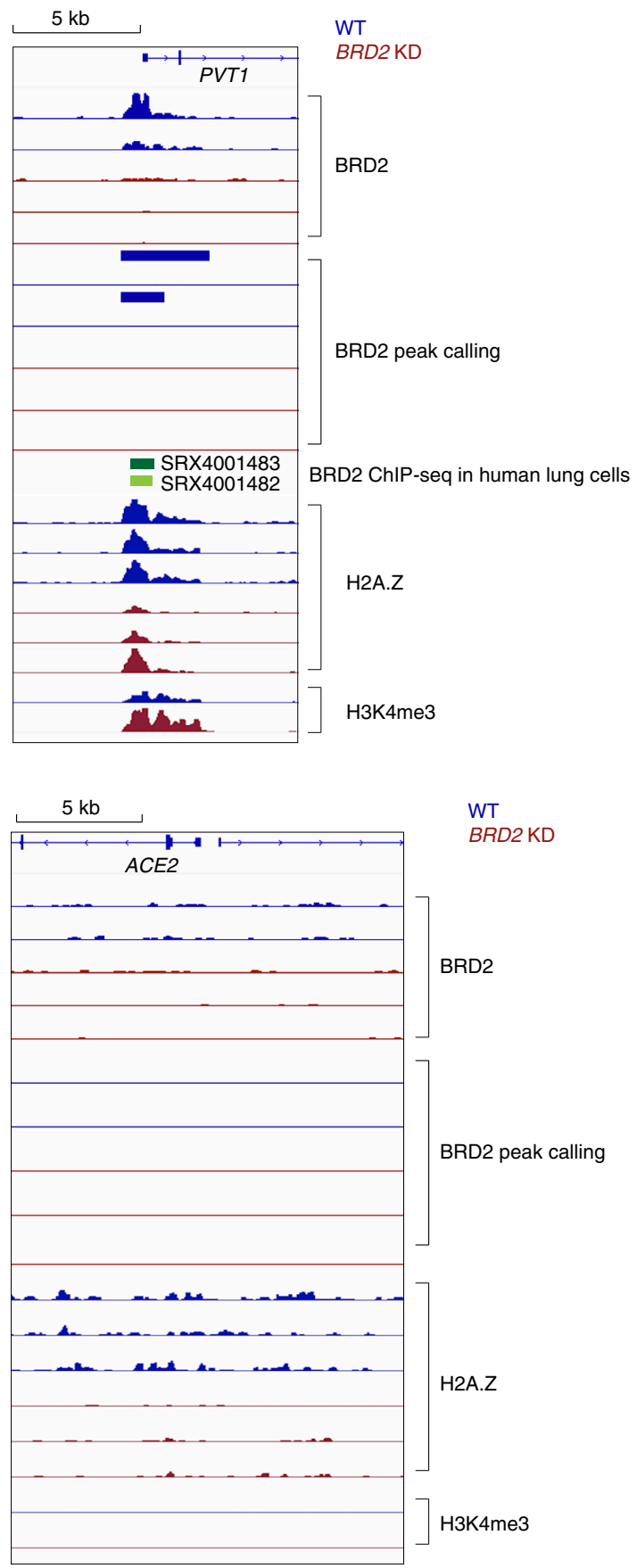

b

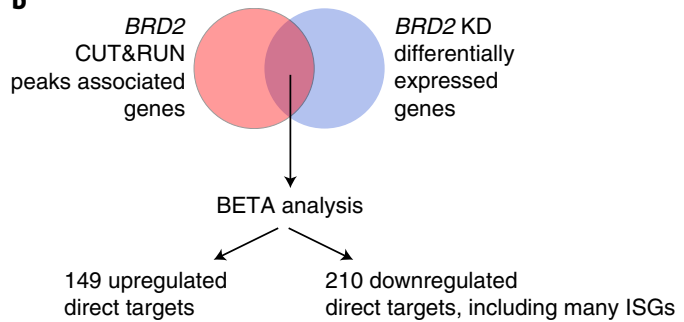

Interferon- $\alpha / \beta$ signalling $P=3.77 \times 10^{-8}$

Interferon signalling $P=7.47 \times 10^{-6}$

Cytokine signalling in immune system $P=3.13 \times 10^{-5}$

Regulation of beta-cell development $P=3.73 \times 10^{-4}$

$\begin{array}{llllllll}0 & 1 & 2 & 3 & 4 & 5 & 6 & 7\end{array}$

d
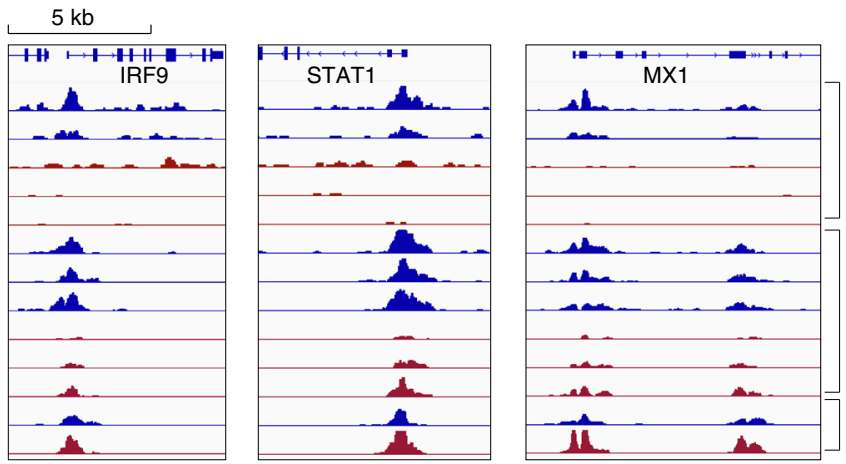

WT

BRD2 KD

BRD2

H2A.Z

H3K4me3

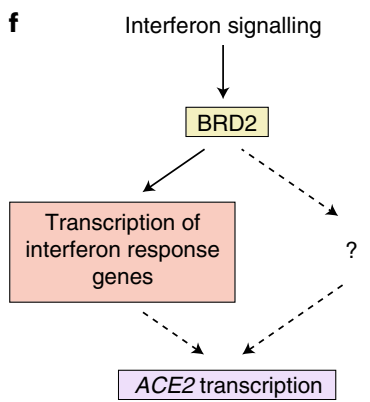



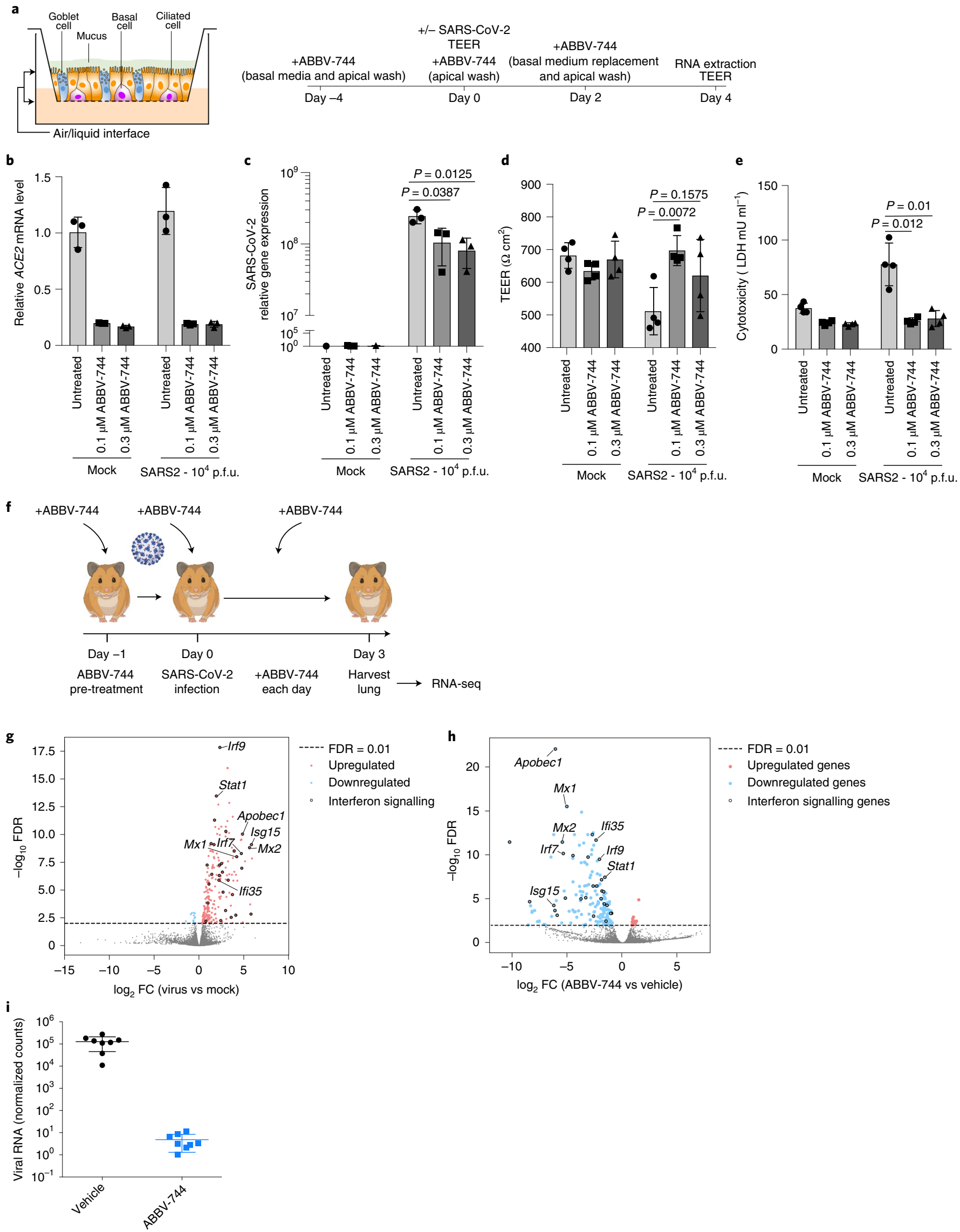

Upregulated genes

Downregulated genes

Interferon signalling genes 
Fig. 6 | BRD2 inhibitors prevent cytotoxicity and reduce SARS-CoV-2 infection in human primary nasal epithelia and inhibit SARS-CoV-2 infection in Syrian hamsters. a, Design for reconstructed human nasal epithelia experiments. b, ACE2 transcript levels relative to the average of GAPDH, TFRC, RPL13 and ACTB as a function of ABBV-744 concentration and/or SARS-CoV-2 infection (right). The average ACE2 mRNA level relative to mock infection and vehicle-treated of $n=3$ biological replicates, with error bars representing the standard deviation, are shown. c, Intracellular SARS-CoV-2 gene expression relative to the average of GAPDH, TFRC, RPL13 and ACTB as a function of ABBV-744 concentration and/or SARS-CoV-2 infection (right). The average of $n=3$ biological replicates and error bars representing the standard deviation are shown. $P$ values were determined using Student's unpaired two-tailed $t$-test. SARS-CoV-2 gene expression relative to mock infection and vehicle-treated are shown. $\mathbf{d}$, Transepithelial electrical resistance (TEER), evaluated as a function of ABBV-744 concentration and/or SARS-CoV-2 infection. The average of $n=4$ biological replicates and error bars representing the standard deviation are shown. $P$ values were determined using Student's unpaired two-tailed $t$-test. e, Cytotoxicity, as measured by LDH release, evaluated as a function of ABBV-744 concentration and/or SARS-CoV- 2 infection. The average of $n=4$ biological replicates and error bars representing the standard deviation are shown. $P$ values were determined using Student's unpaired two-tailed $t$-test. $\mathbf{f}$, Experimental design for the Syrian hamster experiments. g, Volcano plot showing differentially expressed genes for Syrian hamster lungs infected or not with SARS-CoV-2. A subset of ISGs for which BRD2 peaks were identified by CUT\&RUN are labelled. $\mathbf{h}$, Volcano plot showing differentially expressed genes for Syrian hamster lungs infected with SARS-CoV-2 and treated with vehicle or ABBV-744 at $100 \mathrm{nM}$. A subset of ISGs for which BRD2 peaks were identified by CUT\&RUN are labelled. i, Normalized viral RNA counts for Syrian hamsters infected with SARS-CoV-2 and treated with vehicle or ABBV-744 at $100 \mathrm{nM}$. The average of $n=8$ biological replicates and error bars representing the standard deviation are shown.

Next, we tested whether ABBV-744 could reduce SARS-CoV-2 infection in golden Syrian hamsters. Syrian hamsters provide a physiologically relevant model for SARS-CoV-2 infection, with high viral replication and signs of lung involvement ${ }^{38-41}$. In our hands, hamsters did not show substantial weight loss following SARS-CoV-2 infection (Extended Data Fig. 6b). After $24 \mathrm{~h}$ of treatment with ABBV-744 or vehicle, hamsters were infected with SARS-CoV-2 (Fig. 6f) and treated daily with ABBV-744 or vehicle. Three days post-infection, the lungs of hamsters were harvested and subjected to RNA-seq. Infected, but untreated, hamsters showed marked upregulation of a number of genes including ISGs when compared to uninfected controls (Fig. 6g). By contrast, infected hamsters treated with ABBV-744 showed a downregulation of ISG (Fig. 6h) levels relative to vehicle-treated infected hamsters, confirming ABBV-744 activity. Remarkably, viral RNA counts were reduced by about five orders of magnitude in the ABBV-744-treated hamsters versus those treated with vehicle controls (Fig. 6i). Thus, Brd2 inhibition can dramatically decrease SARS-CoV-2 infection in Syrian hamsters.

\section{Discussion}

We have demonstrated that BRD2 is necessary for ACE2 expression in a number of different SARS-CoV-2-relevant systems. We also found that treatment with ABBV-744, a bromodomain inhibitor, can reduce SARS-CoV-2 viral RNA concentrations in primary human nasal epithelial cells and Syrian hamsters. These findings suggest that pharmacological BRD2 inhibitors may be of therapeutic benefit to prevent or reduce the impact of SARS-CoV-2 infection.

Our data suggest that BRD2 is an indirect regulator of ACE2 transcription and thus viral entry in COVID-19-relevant cell types. This is consistent with two other recent studies that show that BET-domain inhibitors can prevent SARS-CoV-2 infection in culture and reduce $A C E 2$ gene expression ${ }^{42,43}$. Our data suggest that BRD2 is the most likely target of the BET-domain inhibitors used in these studies, as opposed to other BET-domain containing proteins, such as BRD4.

We also show that BRD2 is required for interferon-mediated stimulation of ACE2 expression, as both exogenous interferon stimulation and basal interferon stimulation of ACE2 expression are blocked upon BRD2 knockdown or pharmacological inhibition (Fig. 6i). Indeed, inhibition of the Janus kinase (required for transduction of interferon signalling) with ruxolitinib has been shown to downregulate $A C E 2$ levels in cell culture ${ }^{44}$. This does not, however, preclude a more direct, and interferon-independent, regulatory mode.

Our data also show that BRD2 activity is essential for the transcription of ISGs in cell culture and in Syrian hamsters. Based on our findings and the previous literature ${ }^{36}, \mathrm{BRD} 2$ regulation of ISG transcription is probably mediated by a reduction in histone $\mathrm{H} 2 \mathrm{~A} . \mathrm{Z}$ occupancy at these promoters. Intriguingly, these BRD2-dependent effects might be cell-type specific, as one study ${ }^{45}$ has shown that BET inhibition, but not the BET inhibitor ABBV-744, can block cardiac dysfunction due to inflammation caused by SARS-CoV-2 infection. Either way, our data indicate that BRD2 could be a key regulator of the host response to SARS-CoV-2 infection.

The previously described ${ }^{32}$ interaction between the SARS-CoV-2 E protein and BRD2 might have evolved to manipulate gene expression during infection, including the expression of ACE2. In isolation, however, protein $\mathrm{E}$ overexpression in Calu-3 cells did not recapitulate expression changes resulting from BRD2 knockdown or inhibition (Fig. 4a). These data suggest that there is no direct effect of protein E on BRD2 function, or that other viral or host factors expressed during SARS-CoV-2 infection are required to modulate BRD2 function. Further studies are needed to define the function of the protein E-BRD2 interaction.

Several previous CRISPR screens aiming to uncover strategies to inhibit SARS-CoV-2 infection were carried out in cell lines in which an ACE2 transgene was overexpressed ${ }^{9,10}$. Those screens therefore failed to uncover BRD2 as a regulator of endogenous ACE2 expression. BRD2 did show a phenotype, however, in a CRISPR screen carried out in Vero-E6 cells (which express ACE2 endogenously) ${ }^{12}$, although it was not further characterized in that study. These differences highlight the importance of conducting CRISPR-based screens in disease-relevant cell types.

There is a growing literature about the relationship between COVID-19 disease severity, ACE2 expression and interferon regulation $^{1-6}$. Given that ACE2 is known to promote recovery after lung injury and that SARS-CoV-2 manipulates the host interferon response $\mathrm{e}^{46-48}$, the misregulation of these two pathways may play a major role in enhancing the severity of COVID-19. Our data suggest that BRD2 is central to this regulatory network and therefore pharmacological targeting of BRD2 may be a promising therapeutic strategy for the treatment of COVID-19: BRD2 inhibition could both block viral entry, through ACE2 downregulation, and act as an 'emergency brake' for misregulated patient immune responses to COVID-19, via downregulation of ISGs.

\section{Online content}

Any methods, additional references, Nature Research reporting summaries, source data, extended data, supplementary information, acknowledgements, peer review information; details of author contributions and competing interests; and statements of data and code availability are available at https://doi.org/10.1038/ s41556-021-00821-8. 
Received: 18 January 2021; Accepted: 24 November 2021; Published online: 13 January 2022

\section{References}

1. Ziegler, C. G. K. et al. SARS-CoV-2 receptor ACE2 is an interferon-stimulated gene in human airway epithelial cells and is detected in specific cell subsets across tissues. Cell 181, 1016-1035 (2020).

2. Chu, H. et al. Comparative replication and immune activation profiles of SARS-CoV-2 and SARS-CoV in human lungs: an ex vivo study with implications for the pathogenesis of COVID-19. Clin. Infect. Dis. 71, 1400-1409 (2020).

3. Gutiérrez-Chamorro, L. et al. SARS-CoV-2 infection suppresses ACE2 function and antiviral immune response in the upper respiratory tract of infected patients. Preprint at bioRxiv https://doi.org/10.1101/2020.11. 18.388850 (2020).

4. Blanco-Melo, D. et al. Imbalanced host response to SARS-CoV-2 drives development of COVID-19. Cell 181, 1036-1045 (2020).

5. Bastard, P. et al. Autoantibodies against type I IFNs in patients with life-threatening COVID-19. Science 370, eabd4585 (2020).

6. Hadjadj, J. et al. Impaired type I interferon activity and inflammatory responses in severe COVID-19 patients. Science 369, 718-724 (2020).

7. Zhang, Q. et al. Inborn errors of type I IFN immunity in patients with life-threatening COVID-19. Science 370, eabd4570 (2020).

8. Samuel, R. M. et al. Androgen signaling regulates SARS-CoV-2 receptor levels and is associated with severe COVID-19 symptoms in men. Cell Stem Cell 27, 876-889 (2020).

9. Daniloski, Z. et al. Identification of required host factors for SARS-CoV-2 infection in human cells. Cell 184, 92-105 (2020).

10. Wang, R. et al. Genetic screens identify host factors for SARS-CoV-2 and common cold coronaviruses. Cell 184, 106-119.e14 (2020).

11. Schneider, W. M. et al. Genome-scale identification of SARS-CoV-2 and pan-coronavirus host factor networks. Cell 184, 120-132 (2021).

12. Wei, J. et al. Genome-wide CRISPR screens reveal host factors critical for SARS-CoV-2 infection. Cell 184, 76-91 (2020).

13. Shi, J. \& Vakoc, C. R. The mechanisms behind the therapeutic activity of BET bromodomain inhibition. Mol. Cell 54, 728-736 (2014).

14. Fujisawa, T. \& Filippakopoulos, P. Functions of bromodomain-containing proteins and their roles in homeostasis and cancer. Nat. Rev. Mol. Cell Biol. 18, 246-262 (2017).

15. Lui, I. et al. Trimeric SARS-CoV-2 Spike interacts with dimeric ACE2 with limited intra-Spike avidity. Preprint at bioRxiv https://doi.org/10.1101/2020. 05.21.109157 (2020).

16. Lan, J. et al. Structure of the SARS-CoV-2 Spike receptor-binding domain bound to the ACE2 receptor. Nature 581, 215-220 (2020).

17. Chua, R. L. et al. COVID-19 severity correlates with airway epitheliumimmune cell interactions identified by single-cell analysis. Nat. Biotechnol. $\mathbf{3 8}$ 970-979 (2020).

18. Tseng, C.-T. K. et al. Apical entry and release of severe acute respiratory syndrome-associated coronavirus in polarized Calu-3 lung epithelial cells. $J$. Virol. 79, 9470-9479 (2005).

19. Kuchi, S., Gu, Q., Palmarini, M., Wilson, S. J. \& Robertson, D. L. Meta-analysis of virus-induced host gene expression reveals unique signatures of immune dysregulation induced by SARS-CoV-2. Preprint at bioRxiv https://doi.org/10.1101/2020.12.29.424739 (2020).

20. Gilbert, L. A. et al. CRISPR-mediated modular RNA-guided regulation of transcription in eukaryotes. Cell 154, 442-451 (2013).

21. Gilbert, L. A. et al. Genome-scale CRISPR-mediated control of gene repression and activation. Cell 159, 647-661 (2014).

22. Horlbeck, M. A. et al. Compact and highly active next-generation libraries for CRISPR-mediated gene repression and activation. eLife 5, e19760 (2016).

23. Deffieu, M. S. et al. Rab7-harboring vesicles are carriers of the transferrin receptor through the biosynthetic secretory pathway. Sci. Adv. 7, 1-17 (2021).

24. Doroshow, D. B., Eder, J. P. \& LoRusso, P. M. BET inhibitors: a novel epigenetic approach. Ann. Oncol. 28, 1776-1787 (2017).
25. Xu, Y. \& Vakoc, C. R. Targeting cancer cells with BET bromodomain inhibitors. Cold Spring Harb. Perspect. Med. 7, a026674 (2017).

26. Filippakopoulos, P. et al. Selective inhibition of BET bromodomains. Nature 468, 1067-1073 (2010).

27. Faivre, E. J. et al. Selective inhibition of the BD2 bromodomain of BET proteins in prostate cancer. Nature 578, 306-310 (2020).

28. Winter, G. E. et al. BET bromodomain proteins function as master transcription elongation factors independent of CDK9 recruitment. Mol. Cell 67, 5-18 (2017).

29. Shi, C. et al. PROTAC induced-BET protein degradation exhibits potent anti-osteosarcoma activity by triggering apoptosis. Cell Death Dis. 10, 815 (2019).

30. Bermejo, J. A. P. et al. SARS-CoV-2 infection of human iPSC derived cardiac cells reflects cytopathic features in hearts of patients with COVID-19. Sci. Transl. Med. 13, 1-15 (2021).

31. Mulay, A. et al. SARS-CoV-2 infection of primary human lung epithelium for COVID-19 modeling and drug discovery. Cell Rep. 35, 109055 (2021).

32. Gordon, D. E. et al. A SARS-CoV-2 protein interaction map reveals targets for drug repurposing. Nature 583, 459-468 (2020).

33. Skene, P. J. \& Henikoff, S. An efficient targeted nuclease strategy for high-resolution mapping of DNA binding sites. eLife 6, e21856 (2017).

34. Handoko, L. et al. JQ1 affects BRD2-dependent and independent transcription regulation without disrupting H4-hyperacetylated chromatin states. Epigenetics 13, 410-431 (2018).

35. Wang, S. et al. Target analysis by integration of transcriptome and ChIP-seq data with BETA. Nat. Protoc. 8, 2502-2515 (2013).

36. Au-Yeung, N. \& Horvath, C. M. Histone H2A.Z suppression of interferon-stimulated transcription and antiviral immunity is modulated by GCN5 and BRD2. iScience 6, 68-82 (2018).

37. Robinot, R. et al. SARS-CoV-2 infection induces the dedifferentiation of multiciliated cells and impairs mucociliary clearance. Nat. Commun. 12, 4354 (2021)

38. Osterrieder, N. et al. Age-dependent progression of SARS-CoV-2 infection in Syrian hamsters. Viruses 12, 94301 (2020).

39. Imai, M. et al. Syrian hamsters as a small animal model for SARS-CoV-2 infection and countermeasure development. Proc. Natl Acad. Sci. USA 117, 16587-16595 (2020).

40. Sia, S. F. et al. Pathogenesis and transmission of SARS-CoV-2 in golden hamsters. Nature 583, 834-838 (2020).

41. Rosenke, K. et al. Defining the Syrian hamster as a highly susceptible preclinical model for SARS-CoV-2 infection. Emerg. Microbes Infect. 9, 2673-2684 (2020).

42. Qiao, Y. et al. Targeting transcriptional regulation of SARS-CoV-2 entry factors ACE2 and TMPRSS2. Proc. Natl Acad. Sci. USA 118, e2021450118 (2020).

43. Gilham, D. et al. Bromodomain and extraterminal protein inhibitor, apabetalone (RVX-208), reduces ACE2 expression and attenuates SARS-CoV-2 infection in vitro. Biomedicines 9, 437 (2021).

44. Lee, H. K., Jung, O. \& Hennighausen, L. JAK inhibitors dampen activation of interferon-stimulated transcription of ACE2 isoforms in human airway epithelial cells. Commun. Biol. 4, 654 (2021)

45. Mills, R. J. et al. BET inhibition blocks inflammation-induced cardiac dysfunction and SARS-CoV-2 infection. Cell 184, 2167-2182 (2021).

46. Ribero, M. S., Jouvenet, N., Dreux, M. \& Nisole, S. Interplay between SARS-CoV-2 and the type I interferon response. PLoS Pathog. 16, e1008737 (2020).

47. Lei, X. et al. Activation and evasion of type I interferon responses by SARS-CoV-2. Nat. Commun. 11, 3810 (2020).

48. Xia, H. et al. Evasion of type I interferon by SARS-CoV-2. Cell Rep. 33, $108234(2020)$

Publisher's note Springer Nature remains neutral with regard to jurisdictional claims in published maps and institutional affiliations.

(c) The Author(s), under exclusive licence to Springer Nature Limited 2022 


\section{Methods}

Cell culture. Calu-3 cells were cultured in RPMI 1640 (Life Technologies, 22400105 ) with $10 \%$ fetal bovine serum (FBS; VWR, $89510-186$ ), $1 \%$ pen/strep (Life Technologies, 15140122) and $5 \mathrm{mM}$ glutamine (Life Technologies, 25030081) at $37^{\circ} \mathrm{C}$ and $5 \% \mathrm{CO}_{2}$. Cells were split by treatment with TrypLE (Life Technologies, 12604013 ) for $15 \mathrm{~min}$, quenching with media and spinning down at $200 \mathrm{~g}$ for $5 \mathrm{~min}$

At Institut Pasteur, where virus infections were carried out, Calu-3 cells were cultured in minimum essential medium (MEM; Gibco, 11095-080) with 20\% FBS (Gibco, A3160801), 1\% pen/strep (Gibco, 15140-122), 1\% non-essential amino acids (NEAA; Sigma-Aldrich, M7145) and $1 \mathrm{mM}$ sodium pyruvate (Sigma-Aldrich, S8636). These were split in trypsin-EDTA 0.05\% (Gibco, 11580626).

HEK293 cell culture and the production of lentivirus were performed as previously described ${ }^{49}$.

A vial of short tandem repeat (STR) authenticated Caco-2 cells was obtained from the UCSF Cell and Genome Engineering Core (CGEC). Caco-2 cells were cultured in Eagle's minimum essential medium (EMEM; ATCC, 30-2003) with 20\% FBS (VWR. 89510-186), 1\% pen/strep (Life Technologies, 15140122) and $5 \mathrm{mM}$ glutamine (Life Technologies, 25030081) at $37^{\circ} \mathrm{C}$ and $5 \% \mathrm{CO}_{2}$

A vial of A549 cells was obtained from D. Ruggero's laboratory as a gift. A549 cells were cultured in Dulbecco's modified Eagle medium (DMEM; Thermo Fisher Scientific, 10313-039) with 10\% FBS (VWR, 89510-186), 1\% pen/strep (Life Technologies, 15140122) and $5 \mathrm{mM}$ glutamine (Life Technologies, 25030081) at $37^{\circ} \mathrm{C}$ and $5 \% \mathrm{CO}_{2}$

Human iPSC-derived cardiomyocytes were generated and cultured, as previously described ${ }^{30}$, from AICS90 iPSCs (Allen Institute Cell catalogue). Drugs were added on day 69 of differentiation, and cardiomyocytes were collected for analysis on day 72 .

Normal human bronchial epithelia (Mattek NHBE-CRY) were cultured following the supplier's instructions.

Generation of the Calu-3 ACE2 knockout line. The polyclonal ACE2 knockout Calu-3 cell line was generated using the Gene KO kit V2 from Synthego, using three sgRNAs targeting ACE2 with the following protospacer sequences: sRNA1, 5'-GACAUUCUCUUCAGUAAUAU-3'; sgRNA2, 5' -AAACUUGUCCAAAAAUGUCU-3'; sgRNA3,

5'-UUACAGCAACAAGGCUGAGA-3'. The sgRNAs were designed according to Synthego's multiguide gene knockout kit ${ }^{50}$. Briefly, two or three sgRNAs are bioinformatically designed to work in a cooperative manner to generate small, knockout-causing fragment deletions in early exons. These fragment deletions are larger than standard indels generated from single guides. The genomic repair patterns from a multiguide approach are highly predictable on the basis of the guide spacing and design constraints to limit off-targets, resulting in a higher-probability protein knockout phenotype.

The ribonucleoprotein complex with a ratio of 4.5:1 of sgRNA and Cas9 was delivered following the protocol of the SE Cell Line 4D-NucleofectorTM X Kit (Lonza, V4XC-1012), using the nucleofection program DS-130 on the Lonza 4D $\mathrm{X}$ unit. At $72 \mathrm{~h}$ post-transfection, genomic DNA was extracted to serve as the template for PCR amplification of the region that covers the sites targeted by the sgRNAs with the following two primers: ACE2-F, 5'-CTGGGACTCCAAAATCA GGGA-3'; ACE2-R, 5'-CGCCCAACCCAAGTTCAAAG-3'. Sanger sequencing reactions using the sequencing primer ACE2-seq $5^{\prime}$-CAAAATCAGGGATATGG AGGCAAACATC- $3^{\prime}$ were then performed, and the knockout efficiency was determined to be $80 \%$ with ICE software from Synthego ${ }^{51}$ (v2, https://ice.synthego. com/\#/).

Generation of the Calu-3 CRISPRi line. The parental Calu-3 line was obtained from the UCSF Cell and Genome Engineering Core. Calu-3 cells were cultured at $37^{\circ} \mathrm{C}$ with $5 \% \mathrm{CO}_{2}$ in EMEM medium containing $10 \% \mathrm{FBS}, 100 \mathrm{U} \mathrm{ml}^{-1}$ streptomycin, $100 \mu \mathrm{g} \mathrm{ml}^{-1}$ penicillin and $2 \mathrm{mM}$ glutamine. To generate the CRISPRi lines, $\sim 3 \times 10^{6}$ cells were seeded into medium containing lentiviral particles packaging dCas9-BFP-KRAB under a UCOE (universal chromatin opening element)-SFFV (spleen focus-forming virus) promoter $^{52}$. Five days post-infection, blue fluorescent protein (BFP)-positive cells were sorted using BD Fusion. To validate the CRISPRi line, Calu-3-CRISPRi cells were transduced with lentiviral particles expressing non-targeting sgRNA (protospacer $5^{\prime}$-GCTCCCAGTCGGC ACCACAG-3') or CD81-targeting sgRNA (protospacer 5'-GGCCTGGCAGGATG CGCGG-3'). CD81 expression was measured seven days post-transduction by dislodging cells with TrypLE and live cells were stained with Allophycocyanin (APC)-conjugated anti-human CD81 antibody (Biolegend, 349509). CD81 expression was assessed on a BD LSRII system with $>90 \%$ of Calu-3 CRISPRi cells with CD81 knocked down compared to a non-targeting sgRNA control.

Spike-RBD binding assay. Recombinant biotinylated SARS-CoV-2 spike Spike-receptor-binding domain with a C-terminal human IgG Fc domain fusion (referred to as Spike-RBD) was prepared as previously described ${ }^{6}$. Calu- 3 cells were grown in 96-well flat-bottom plates until $>50 \%$ confluence. Medium was aspirated and cells were washed once with phosphate-buffered saline (PBS). Cells were then treated with TrypLE to release them from the plate, RPMI 1640 medium was added to dilute TrypLE, and cells were pelleted by centrifugation at $200 \mathrm{~g}$ for $5 \mathrm{~min}$. From this point on, all steps were carried out on ice. Cells were incubated in 3\% bovine serum albumin (BSA; Sigma-Aldrich, A7030) in Dulbecco's phosphate-buffered saline (DPBS; Sigma-Aldrich, D8537) for 15 min to block, then washed twice in 3\% BSA in DPBS by centrifugation at $200 \mathrm{~g}$ for $5 \mathrm{~min}$ in V-bottom plates, followed by resuspension. Spike-RBD was diluted in 3\% BSA to the appropriate concentrations and incubated with cells for $30 \mathrm{~min}$ on ice. Cells were then washed twice with $3 \%$ BSA in DPBS and incubated with anti-strep PE-Cy7 (Thermo Fisher, SA1012) at $5 \mu \mathrm{g} \mathrm{ml}^{-1}$. Cells were washed twice and subjected to flow cytometry on a FACSCelesta in HTS mode. Cells were gated to exclude doublets and the median PE-Cy7 signal was calculated for each sample. The gating strategy is shown in Supplementary Fig. 2. BD FACSDiva (version 8.01.1) was used to collect flow cytometry data and perform fluorescence activated cell sorting (FACS). $\mathrm{EC}_{50}$ values and their $95 \%$ confidence intervals were calculated by fitting the RBD binding data into a Sigmoidal 4PL model in Prism 6.

CRISPRi screen. Calu- 3 cells were infected with the H1 CRISPRi sgRNA library ${ }^{22}$ as described in ref. ${ }^{49}$ and selected using treatment with $1 \mu \mathrm{g} \mathrm{ml}^{-1}$ puromycin for 3 days. After selection, cells were stained with $10 \mathrm{nM}$ Spike-RBD as described above or for TFRC as previously described ${ }^{49}$ and subjected to FACS, where cells were sorted into the top $30 \%$ and bottom $30 \%$ based on high and low expression of TFRC or Spike-RBD. Because of the viability and stickiness known for Calu-3 cells, coverage was lower than optimal, at 200 -fold over the library diversity. Sorted populations were spun down at $200 \mathrm{~g}$ for $5 \mathrm{~min}$, and genomic DNA was isolated as described in ref. ${ }^{49}$. sgRNA cassettes were amplified by PCR and sequencing and analysis was performed as described in ref. ${ }^{49}$ but with FDR of 0.1 rather than 0.05 or 0.01 , due to noise.

Validation of screening hits. Individual sgRNAs were selected based on phenotypes in the primary screens and cloned into a lentiviral expression vector as described ${ }^{49}$. Protospacer sequences of these sgRNAs are provided in cells expressing sgRNAs were selected using treatment with $1 \mu \mathrm{g} \mathrm{ml}^{-1}$ puromycin for 3-7 days.

Drug treatments. Drugs (ABBV-744, Selleckchem, S8723; JQ1, Sigma-Aldrich, SML1524; dBET-6, Selleckchem, S8762) were dissolved in dimethyl sulfoxide (DMSO) or water according to the manufacturers' instructions. Cells were treated with drugs for $72 \mathrm{~h}$ with medium changes performed every $24 \mathrm{~h}$ with medium containing fresh drug.

Interferon treatments. IFN $\beta$ (R\&D Systems, $8499-$ IF) was dissolved according to the manufacturer's instructions. Cells were treated with IFN- $\beta$ for $72 \mathrm{~h}$, with medium changes performed every $24 \mathrm{~h}$ with medium containing fresh IFN- $\beta$.

Quantitative PCR. qPCR was performed and analysed as described in ref. ${ }^{49}$. Primers: ACE2 forward, GGTCTTCTGTCACCCGATTT; ACE2 reverse, CATC CACCTCCACTTCTCTAAC; ACTB forward, ACCTTCTACAATGAGCTGCG; ACTB reverse, CCTGGATAGCAACGTACATGG; IRF9 forward, GCCCTACAA GGTGTATCAGTTG; IRF9 reverse, TGCTGTCGCTTTGATGGTACT; IFNAR1 forward, AACAGGAGCGATGAGTCTGTC; IFNAR1 reverse, TGCGAAATGG TGTAAATGAGTCA; STAT1 forward, CAGCTTGACTCAAAATTCCTGGA; STAT1 reverse, TGAAGATTACGCTTGCTTTTCCT

Western blotting. Cells from one confluent well of a six-well plate were lysed in RIPA buffer plus cOmplete EDTA-free protease inhibitor tablets (Roche, 11873580001 ) and spun for $10 \mathrm{~min}$ at $21,000 \mathrm{~g}$ at $4^{\circ} \mathrm{C}$. The pellet was removed and a bicinchoninic acid (BCA) assay (Thermo Fisher, 23225) was performed on the remaining supernatant. Lysate volumes with equivalent protein content were diluted with sodium dodecyl sulfate polyacrylamide gel electrophoresis (SDSPAGE) loading dye and subjected to gel electrophoresis on 4-12\% BisTris SDSPAGE gels (Life Technologies, NP0322). Gels were then transferred and blocked in $5 \%$ non-fat dry milk (NFDM) for $1 \mathrm{~h}$ at room temperature. Antibodies in fresh 5\% NFDM were added (mouse monoclonal GAPDH 1:10,000; goat polyclonal ACE2, R\&D Tech AF933, 1:200; rabbit monoclonal BRD2, Abcam 197865, 1:5,000) and incubated at $4{ }^{\circ} \mathrm{C}$ for at least $16 \mathrm{~h}$. Membranes were washed four times with TBS $+0.1 \%$ Tween- 20 and incubated with secondary antibodies (1:10,000 donkey anti-goat-800 (LI-COR, 926-32214), donkey anti-mouse-680 (LI-COR, 926-68072) and horse radish peroxidase (HRP) donkey anti-rabbit (CST, 7074P2)). Membranes were visualized using a LI-COR or Femto HRP kit (Thermo Fisher, 34094). Uncropped images of western blots are provided as Supplementary Fig. 1.

Virus. The SARS-CoV-2 strain used (BetaCoV/France/IDF0372/2020 strain) was propagated once in Vero-E6 cells and was a gift from the National Reference Centre for Respiratory Viruses at Institut Pasteur, Paris, originally supplied through the European Virus Archive goes global platform.

Cytotoxicity measurements of Calu-3 cells. Thirty-thousand Calu-3 cells per well were seeded into Greiner 96-well white-bottom plates and incubated for $48 \mathrm{~h}$ at $37^{\circ} \mathrm{C}$ and $5 \% \mathrm{CO}_{2}$. The cells were then treated with identical drug concentrations as in the infection assays, for five days, by refreshing the medium with $100 \mu \mathrm{l}$ per 
well fresh drug-containing medium every $24 \mathrm{~h}$. Cell viability was then assayed by adding $100 \mu \mathrm{l}$ per well of CellTiter-Glo 2.0 (Promega) and incubated for $10 \mathrm{~min}$ at room temperature. Luminescence was recorded with an Infinite 200 Pro plate reader (Tecan) using an integration time of $1 \mathrm{~s}$

Virus infection assays. Thirty-thousand Calu- 3 cells per well were seeded into 96-well plates and incubated for $48 \mathrm{~h}$ at $37^{\circ} \mathrm{C}$ and $5 \% \mathrm{CO}_{2}$. At the time of infection, the medium was replaced with virus inoculum (multiplicity of infection of 0.1 plaque-forming units (p.f.u.) per cell) and incubated for $1 \mathrm{~h}$ at $37^{\circ} \mathrm{C}$ and $5 \% \mathrm{CO}_{2}$. Following the 1 -h adsorption period, the inoculum was removed, replaced with fresh medium, and the cells incubated at $37^{\circ} \mathrm{C}$ and $5 \% \mathrm{CO}_{2}$. At $24 \mathrm{~h}, 48 \mathrm{~h}$ and $72 \mathrm{~h}$ post-infection, the cell-culture supernatant was collected, and the viral load was assessed by RT-qPCR as described previously ${ }^{32}$. Briefly, the cell-culture supernatant was collected, heat-inactivated at $95^{\circ} \mathrm{C}$ for 5 min, and used for RT-qPCR analysis. SARS-CoV-2-specific primers targeting the N gene region-5'-TAATCAGACAAGGAACTGATTA-3' (forward) and $5^{\prime}$-CGAAGGTGTGACTTCCATG-3' (reverse) - were used with the Luna Universal One-Step RT-qPCR Kit (New England Biolabs) in an Applied Biosystems QuantStudio 6 thermocycler or an Applied Biosystems StepOnePlus system, with the following cycling conditions: $55^{\circ} \mathrm{C}$ for $10 \mathrm{~min}, 95^{\circ} \mathrm{C}$ for $1 \mathrm{~min}$, and $40 \mathrm{cycles}$ of $95^{\circ} \mathrm{C}$ for $10 \mathrm{~s}$, followed by $60^{\circ} \mathrm{C}$ for $1 \mathrm{~min}$. The number of viral genomes is expressed as p.f.u. equivalents per $\mathrm{ml}$, and was calculated by performing a standard curve with RNA derived from a viral stock with a known viral titre.

Plaque assays. Viruses were quantified by plaque-forming assays. For this, Vero-E6 cells were seeded in 24-well plates at a concentration of $1 \times 10^{5}$ cells per well. The following day, tenfold serial dilutions of individual virus samples in serum-free DMEM medium were added to infect the cells at $37^{\circ} \mathrm{C}$ for $1 \mathrm{~h}$. After the adsorption time, a solid agarose overlay (DMEM, $10 \%$ (vol/vol) PBS and $0.8 \%$ agarose) was added. The cells were incubated for a further three days before fixation with $4 \%$ formalin and visualization using crystal violet solution.

CUT\&RUN. CUT\&RUN was performed with one million Calu-3 cells. Cells were removed from the plate by treatment with Versene (Life Technologies 15040066) for $20 \mathrm{~min}$ and resuspended in fresh medium. They were then spun down and washed twice with DPBS before proceeding with the CUTANA CUT\&RUN kit (EpiCypher, 14-0050). The experiment was performed with the included IgG and H3K4Me control antibodies and the BRD2 antibody (Abcam 197865) as well as Escherichia coli spike-in DNA according to the kit protocol.

QuantSeq analysis. Raw sequencing reads from QuantSeq were trimmed using Trimmomatic ${ }^{53}$ (v0.39, PMID 24695404) and mapped to the human reference transcriptome (GRCh38, GENCODE Release 36) using Salmon ${ }^{54}$ (v1.3.0) to obtain transcript abundance counts. Gene-level count estimates were obtained using tximport ${ }^{54}$ (v1.18.0) with default settings. Subsequently, differential gene-expression analyses were performed using the glmQLFTest method implemented in the edgeR package ${ }^{54}(\mathrm{v} 3.28 .1)$. Cluster $^{55}$ (v3.0) was used for hierarchical clustering and Java TreeView ${ }^{56}(\mathrm{v} 1.1 .6 \mathrm{r} 4)$ for visualization.

CUT\&RUN analysis. CUT\&RUN analysis was performed as previously described ${ }^{57}$. Briefly, paired-end reads were mapped to the human genome GRCh38 using Bowtie2 (v2.3.4.1) with the options --end-to-end --very-sensitive --no-unal --no-mixed --no-discordant --phred33 -I 10 -X 1000. Sparse enrichment analysis for CUT\&RUN (SEACR ${ }^{58}$, https://seacr.fredhutch.org/) was used for peak calling. $\mathrm{H} 3 \mathrm{~K} 4 \mathrm{me} 3$ and BRD2 peaks were normalized to IgG control. Published BRD2 ChIP-seq data in human lung cells were obtained from ChIP-Atlas (https:// chip-atlas.org/). The Integrative Genomics Viewer (IGV, igv.org) was used for visualization.

SARS-CoV-2 infection of reconstructed human nasal epithelia. MucilAir (https:// www.epithelix.com/products/mucilair), corresponding to reconstructed human nasal epithelium cultures differentiated in vitro for at least four weeks, was purchased from Epithelix. Epithelix obtains donor consent and this study does not involve any human participants. The cultures were generated from pooled nasal tissues obtained from 14 human adult donors. Cultures were maintained in air/liquid interface (ALI) conditions in transwells with $700 \mu \mathrm{l}$ of MucilAir medium (Epithelix) in the basal compartment, and kept at $37^{\circ} \mathrm{C}$ under a $5 \% \mathrm{CO}_{2}$ atmosphere. SARS-CoV-2 infection was performed as previously described ${ }^{37}$. Briefly, the apical side of ALI cultures was washed for $20 \mathrm{~min}$ at $37^{\circ} \mathrm{C}$ in Mucilair medium (+/- drug) to remove mucus. Cells were then incubated with $10^{4}$ p.f.u. of the isolate BetaCoV/France/IDF00372/2020 (EVAg collection, Ref-SKU 014V03890; provided by S. Van der Werf). The viral input was diluted in DMEM medium (+/ - drug) to a final volume $100 \mu \mathrm{l}$, and left on the apical side for $4 \mathrm{~h}$ at $37^{\circ} \mathrm{C}$. Control wells were mock-treated with DMEM (Gibco) for the same duration. Viral inputs were removed by washing twice with $200 \mu$ of PBS ( 5 min at $37^{\circ} \mathrm{C}$ ) and once with $200 \mu \mathrm{l}$ of Mucilair medium $\left(20 \mathrm{~min}\right.$ at $\left.37^{\circ} \mathrm{C}\right)$. The basal medium was replaced every 2-3 days. Apical supernatants were collected every 2-3 days by adding $200 \mu \mathrm{l}$ of Mucilair medium on the apical side, with an incubation of $20 \mathrm{~min}$ at $37^{\circ} \mathrm{C}$ before collection.
For ABBV-774 treatment, cultures were pretreated for four days with $100 \mathrm{nM}$ or $300 \mathrm{nM}$ of the drug. For this pretreatment, ABBV-744 was added to an apical wash at day -4 and to the basal compartment from day -4 to day 0 . The drug was then added on the apical side during viral adsorption at day 0 , and then every $2-3$ days to both the apical wash and the basal compartment throughout the infection.

Transepithelial electrical resistance measurements. The apical side of transwell cultures was washed for $20 \mathrm{~min}$ at $37^{\circ} \mathrm{C}$ in Mucilair medium. The transwells were then transferred in a new 24-well plate and DMEM was added to both the apical $(200 \mu \mathrm{l})$ and basal $(700 \mu \mathrm{l})$ sides. The transepithelial electrical resistance was then measured using an Evom 3 ohm meter (World Precision Instruments).

Lactate dehydrogenase cytotoxicity assay. Diluted culture supernatants (1:25) were pretreated with Triton-X100 $1 \%$ for $2 \mathrm{~h}$ at room temperature for viral inactivation. Lactate dehydrogenase (LDH) dosage was performed using the LDH-Glo cytotoxicity assay kit (Promega) following the manufacturer's instructions. Luminescence was measured using an EnSpire luminometer (Perkin Elmer).

Viral RNA quantification. Apical supernatants were stored at $-80^{\circ} \mathrm{C}$ until thawing and were diluted fourfold in PBS for quantification in a 96-well PCR plate. Supernatants were then inactivated for $20 \mathrm{~min}$ at $80^{\circ} \mathrm{C}$. One microlitre of supernatant was added directly to $4 \mu \mathrm{l}$ of PCR reaction mix for SARS-CoV-2 RNA quantification. PCR was carried out in a final volume of $5 \mu \mathrm{l}$ per reaction in 384-well plates using the Luna Universal Probe One-Step RT-qPCR kit (New England Biolabs) with SARS-CoV-2 N-specific primers (forward, $5^{\prime}$-TAA TCA GAC AAG GAA CTG ATT A-3'; reverse, $5^{\prime}$-CGA AGG TGT GAC TTC CAT G-3') on a QuantStudio 6 Flex thermocycler (Applied Biosystems). Standard curves were established in parallel using purified SARS-CoV-2 viral RNA.

Tissue RNA quantification. ACE2 and SARS-CoV-2 expression levels were quantified in epithelial cells by real-time qPCR. The epithelial cultures were washed in ice-cold PBS and then lysed in $150 \mu \mathrm{l}$ of Trizol reagent (Thermo Fisher Scientific) added to the apical side of the insert for $5 \mathrm{~min}$. RNA was purified using the Direct-zol miniprep kit (ZR2080, Zymo Research). Transcripts of genes of interest (ACE2, SARS-CoV-2 N gene) were amplified in a final volume of $5 \mu \mathrm{l}$ per reaction in 384-well plates using the Luna Universal Probe One-Step RT-qPCR kit (New England Biolabs) on a QuantStudio 6 Flex thermocycler. The RT-qPCR results were normalized to the mean expression of four reference genes (GAPDH, TFRC, $A L A S 1$ and RLP13) to compute relative gene expression, as described previously $^{37}$. The ACE2 primers used were ACE2-For 5' -TGG GAC TCT GCC ATT TAC TTA C-3' and ACE2-Rev 5' -CCA GAG CCT CTC ATT GTA GTC T-3'.

In vivo infections. All animal infections were conducted at the Icahn School of Medicine at Mount Sinai, in the biosecurity level 3 (BSL-3) facility of the Global Health and Emerging Pathogen Institute approved by the Institutional Animal Care and Use Committee at Icahn School of Medicine at Mount Sinai under protocol number IACUC\#20-0743. Six- to eight-week-old male golden Syrian hamsters (Mesocricetus auratus) were purchased from Jackson Laboratories, housed in pairs and fed ad libitum. On the day of infection, animals were anaesthetized by administration of $100 \mu \mathrm{l}$ of a ketamine $\mathrm{HCl}$ /xylazine $(4: 1)$ mix by intraperitoneal injection and infected intranasally with 1,000 p.f.u. of SARS-CoV-2 USA-WA1/2020 diluted in $100 \mu \mathrm{l}$ of PBS. At the indicated time points, animals were treated with $1 \mathrm{ml}$ of ABBV-744 by oral gavage. The drug was prepared fresh daily to a final concentration of $20 \mathrm{mg} \mathrm{kg}^{-1}$ in $0.5 \%$ hydroxypropyl methylcellulose $/ 0.5 \%$ Tween 80 in water. On day 3 after infection, animals received $100 \mu \mathrm{l}$ of a mix of pentobarbital/PBS (1:4) intraperitoneally and, once anaesthetized they were cervically dislocated and lung lobes collected in $1 \mathrm{~m}$ of Trizol reagent. Tissues were homogenized in a tissue lyser for two cycles of $40 \mathrm{~s}$, spun down for $5 \mathrm{~min}$ at $8,000 \mathrm{~g}$, and the supernatants were stored at $-80^{\circ} \mathrm{C}$ for plaque assay or RNA extraction.

RNA extraction. RNA extraction was performed following the instructions from the manufacturer of TRIzol reagent (Invitrogen). Briefly, a 1/5 volume of chloroform was added to the lung supernatants in TRIzol, phases were separated by centrifugation and RNA was precipitated by overnight incubation with isopropanol at $-20^{\circ} \mathrm{C}$. The RNA pellet was washed with ethanol $70 \%$ and resuspended in RNase-free water. RNA was quantified by NanoDrop and resuspended to a final

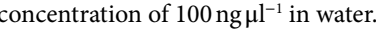

RNA-seq. One microgram of RNA was used as the starting material for library preparation. The kit employed was a TruSeq RNA Library Prep kit v2 (Illumina) over polyadenylated RNA and the manufacturer's instructions were followed. The sequencing was performed on an Illumina NextSeq 500 instrument. The raw reads obtained from the run were aligned against the Syrian golden hamster genome (MesAur1.0) in the BaseSpace platform by Illumina, with the tool 'RNA-Seq Alignment'.

Statistics and reproducibility. All experiments were carried out using standard cell biological/biochemical techniques with pre-validated reagents including 
cell lines. Accordingly, the minimum standard of at least three replicates per experiment was used for all experiments, with the following exceptions. In Fig. la,b we report the dose responses of different cell types to Spike protein where each dose response has data points for seven doses. These experiments were performed once. In Extended Data Fig. 1 we report a dose response with data points for two doses. These experiments were performed once. In Extended Data Fig. 2 we report measured dose responses where each dose response was performed in duplicate and with data points for seven to eight doses. Experiments that were performed with $n=1$ are reported in Figs. $1 \mathrm{c}, \mathrm{e}, \mathrm{f}, 2 \mathrm{c}$ and $3 \mathrm{~b}$. No statistical method was used to predetermine sample size. No data were excluded from analyses. Experiments were not randomized. Investigators were not blinded to allocation during experiments and outcome assessment.

Reporting Summary. Further information on research design is available in the Nature Research Reporting Summary linked to this Article.

\section{Data availability}

Sequencing data are available from the NCBI Gene Expression Omnibus (GEO) with the following accession numbers: GSE165025 (RNA-sequencing data associated with Fig. 4), GSE182993 (CUT\&RUN data associated with Fig. 5) and GSE182994 (RNA-sequencing data associated with Fig. 6f-h). Previously published BRD2 ChIP-seq data that were re-analysed here are available under accession codes GSE113714 and GSE104481. All other data supporting the findings of this study are available from the corresponding author on reasonable request. Source data are provided with this paper.

\section{Code availability}

Analysis of the CRISPRi screen results was carried out using custom code (MAGeCK-iNC) developed in the Kampmann laboratory. This has been described previously ${ }^{49}$ and is freely available at https://kampmannlab.ucsf.edu/mageck-inc.

\section{References}

49. Tian, R. et al. CRISPR interference-based platform for multimodal genetic screens in human iPSC-derived neurons. Neuron 104, 239-255 (2019).

50. Stoner, R., Maures, T. \& Conant, D. Methods and systems for guide RNA design and use US patent application 16/418,893 (2019).

51. Hsiau, T. et al. Inference of CRISPR edits from Sanger trace data. Preprint at bioRxiv https://doi.org/10.1101/251082 (2019).

52. Adamson, B. et al. A multiplexed single-cell CRISPR screening platform enables systematic dissection of the unfolded protein response. Cell 167, 1867-1882 (2016).

53. Bolger, A. M., Lohse, M. \& Usadel, B. Trimmomatic: a flexible trimmer for Illumina sequence data. Bioinformatics 30, 2114-2120 (2014).

54. Patro, R., Duggal, G., Love, M. I., Irizarry, R. A. \& Kingsford, C. Salmon provides fast and bias-aware quantification of transcript expression. Nat. Methods 14, 417-419 (2017)

55. Eisen, M. B., Spellman, P. T., Brown, P. O. \& Botstein, D. Cluster analysis and display of genome-wide expression patterns. Proc. Natl Acad. Sci. USA 95 14863-14868 (1998).
56. Saldanha, A. J. Java Treeview-extensible visualization of microarray data. Bioinformatics 20, 3246-3248 (2004).

57. Skene, P. J., Henikoff, J. G. \& Henikoff, S. Targeted in situ genome-wide profiling with high efficiency for low cell numbers. Nat. Protoc. 13, 1006-1019 (2018).

58. Meers, M. P., Tenenbaum, D. \& Henikoff, S. Peak calling by sparse enrichment analysis for CUT\&RUN chromatin profiling. Epigenet. Chromatin 12, 42 (2019).

\section{Acknowledgements}

We thank members of the Kampmann, Vignuzzi and Conklin laboratories, as well as V. Ramani, D. Ruggero, M. Ott and her laboratory and other members of the UCSF QBI Coronavirus Research Group (QCRG) for helpful discussions. We thank K. Leng for feedback on the manuscript. We acknowledge the Gladstone Stem Cell Core for help with cardiomyocyte production. A.J.S. is supported by NIH grant F32AG063487. G.N.R. is supported by the NSF Graduate Research Fellowship Program (GRFP) and UCSF Discovery Fellowship. S.A.L. was a Merck Fellow of the Helen Hay Whitney Foundation. I.L. was supported by an NSF GRFP award. M.K. is a Chan Zuckerberg Biohub Investigator.

\section{Author contributions}

R.T., A.J.S. and M.K. conceptualized the overall project, analysed the results and prepared the manuscript, with input from all co-authors. V.V.R., A.M.K. and Q.D.T. performed and analysed live-virus experiments in Calu-3 cells, with guidance from M.V. R.R. performed and analysed human nasal epithelia experiments with guidance from L.A.C. L.C. performed and analysed the Syrian hamster experiments, with guidance from B.R.T. G.N.R. and S.J.R. performed and analysed the experiments with cardiomyocytes, with guidance from B.R.C. R.T., A.J.S., M.C. and X.G. performed and analysed all other experiments, with guidance from M.K. J.W. performed and analysed basal interferon signalling knockdown experiments in Calu-3 cells, with guidance from R.T. N.L. analysed the QuantSeq data, with guidance from R.T. S.A.L., I.L. and J.A.W. generated Spike-RBD. J.K.N. and J.S.W. generated the Calu-3 CRISPRi cell line. J.C.-S., J.O., T.M. and K.H. designed and provided sgRNAs to generate the ACE2 knockout cell line.

\section{Competing interests}

J.C.-S., J.O., T.M. and K.H. are employees and shareholders of Synthego Corporation. All other authors declare no competing interests.

\section{Additional information}

Extended data is available for this paper at https://doi.org/10.1038/s41556-021-00821-8. Supplementary information The online version contains supplementary material available at https://doi.org/10.1038/s41556-021-00821-8.

Correspondence and requests for materials should be addressed to Ruilin Tian or Martin Kampmann.

Peer review information Nature Cell Biology thanks Andrew Bowie, Ke Lan and the other, anonymous, reviewers for their contribution to the peer review of this work.

Reprints and permissions information is available at www.nature.com/reprints. 
a

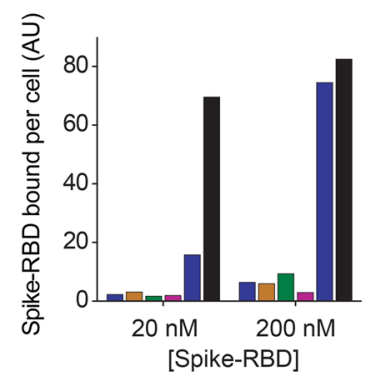

b

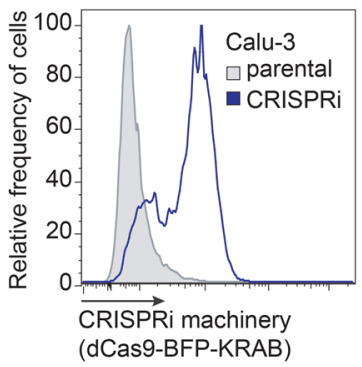

$\%$ of Max

C

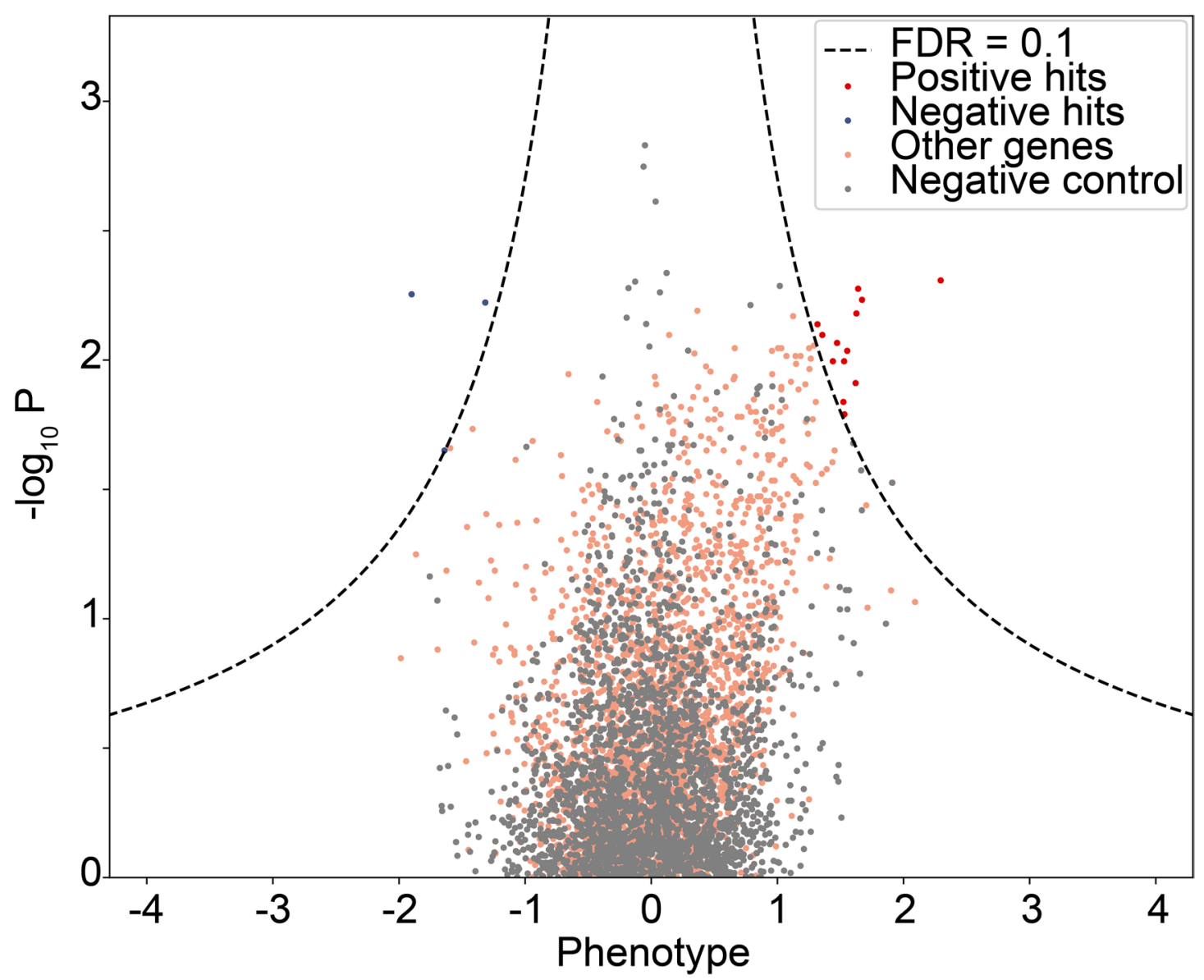

Extended Data Fig. 1 | Calu-3 cells bind Spike-RBD specifically and were engineered to express CRISPRi machinery enabling CRISPRi screening. a, Spike-RBD binding in different cell types at $20 \mathrm{nM}$ and $200 \mathrm{nM}$ Spike-RBD was quantified by flow cytometry. b. Expression of CRISPRi machinery (dCas9-BFP-KRAB) in the CRISPRi Calu-3 line indicated by the expression of BFP by flow cytometry. C, Enrichment of sgRNAs targeting specific genes (coloured dots) or non-targeting control sgRNAs plotted against the negative log of the P-value with a FDR of 0.1 shown (dashed lines). 

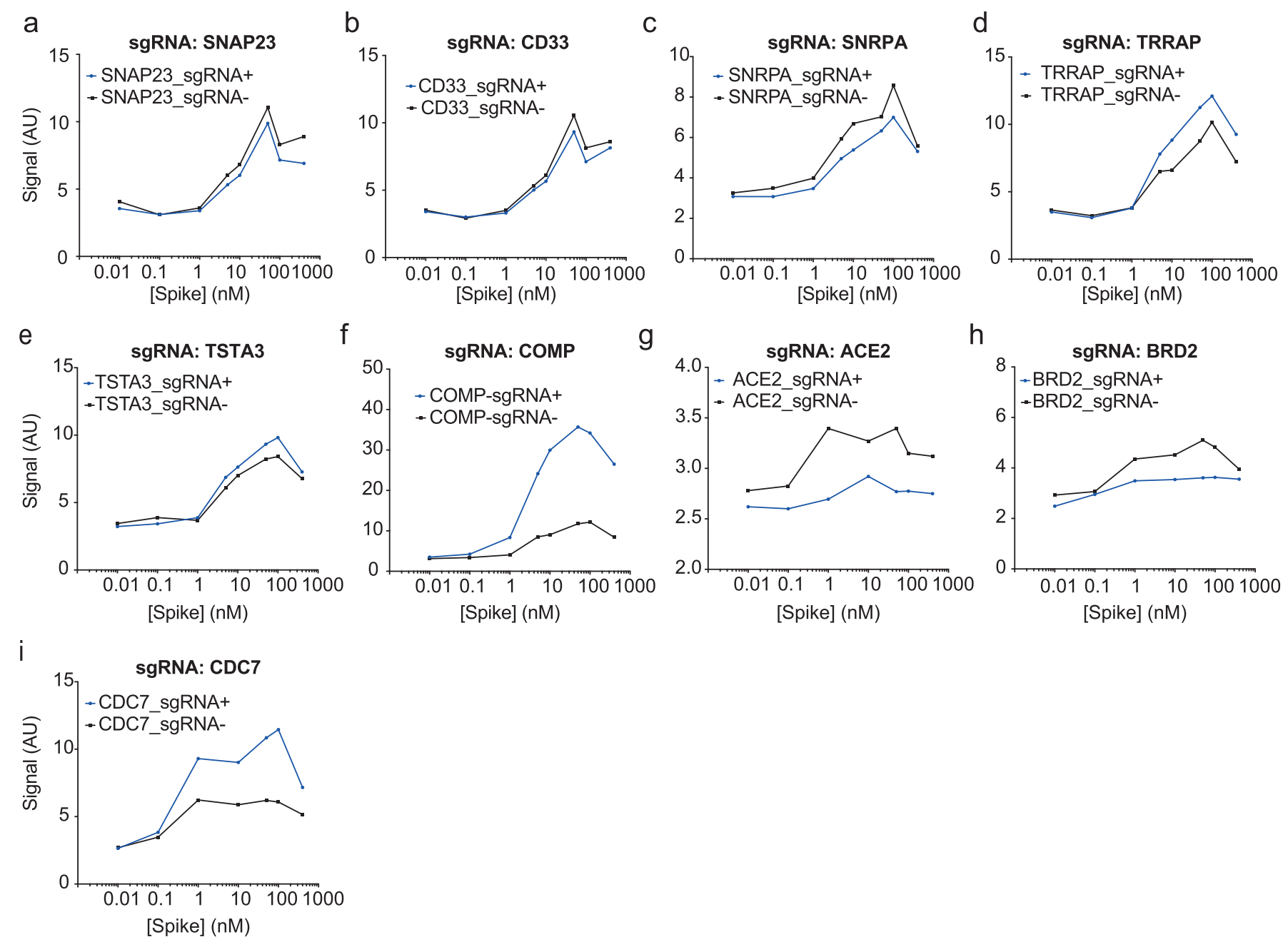

Extended Data Fig. 2 | Individual sgRNA re-test of screening hits. a-i, Spike-RBD signal measured by flow-cytometry as a function of Spike-RBD concentration. Blue lines represent cells expressing the sgRNA targeting the gene of interest, black lines represent un-transduced control cells in the same well. Average of two technical replicates are shown. 


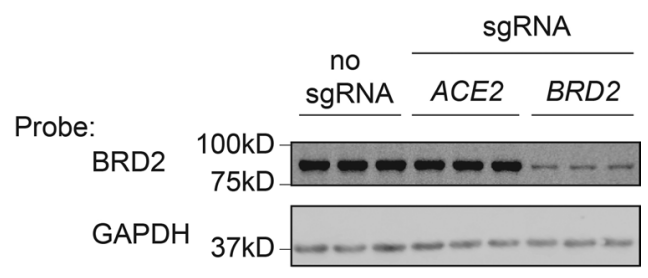

Extended Data Fig. 3 | BRD2 is effectively knocked down by CRISPRi. Western blot for BRD2 and the loading control GAPDH in CRISPRi Calu-3 cells expressing no sgRNA or sgRNAs targeting ACE2 or BRD2. Three lanes represent samples from three independent wells. 
a

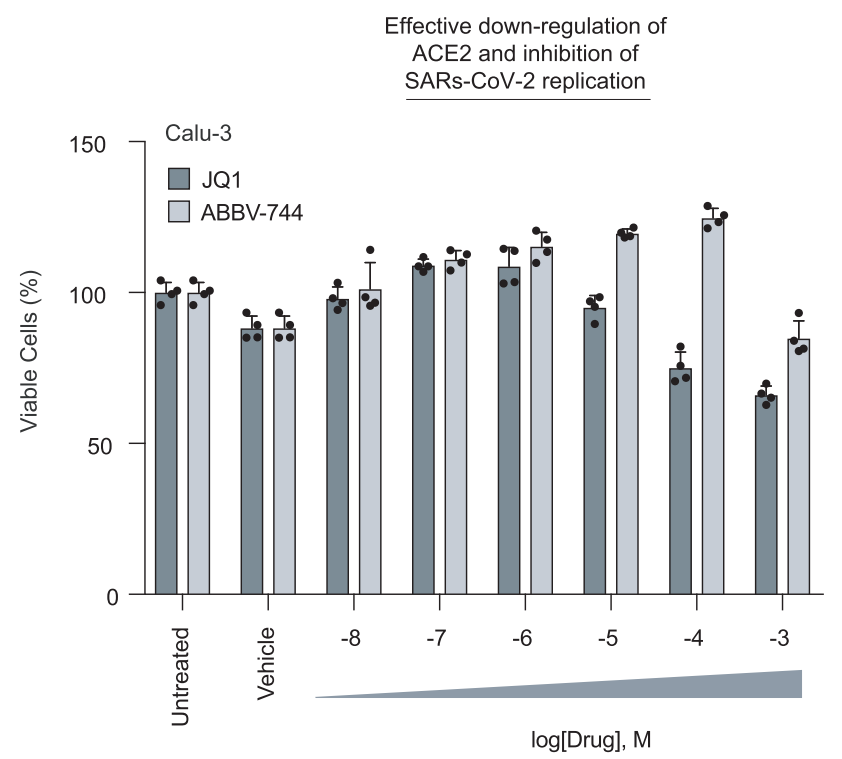

b

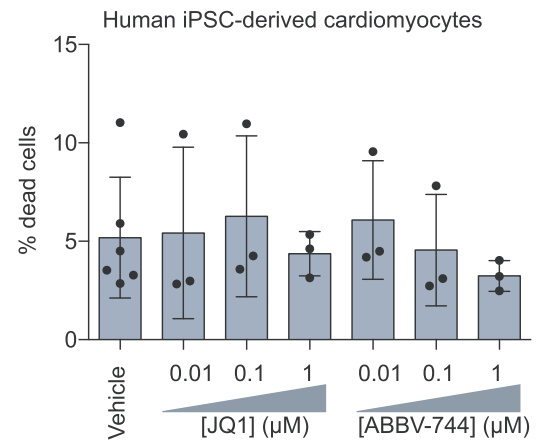

C

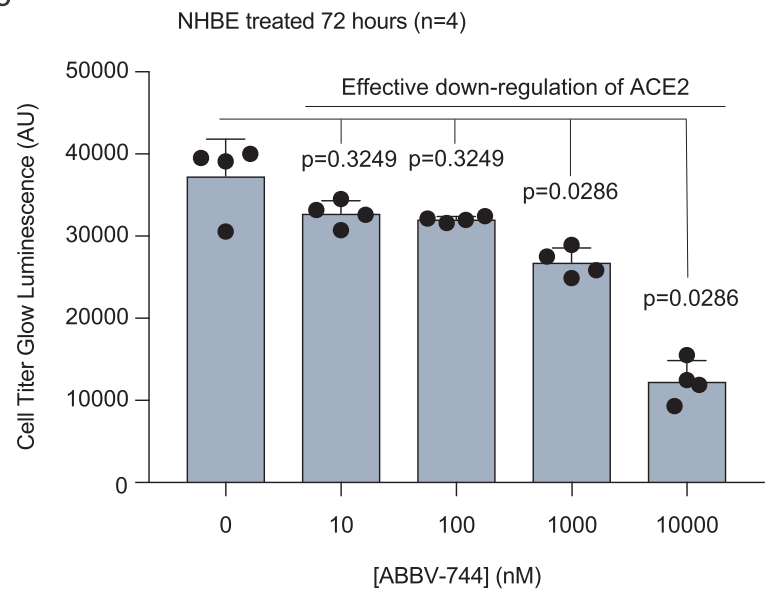

Extended Data Fig. 4 | Non-toxic concentration range of BRD2 inhibitors. a, Calu-3 cells were treated with vehicle or the indicated concentrations of JQ1 or ABBV-744 for 5 days. Cell viability was then assayed with CellTiter-Glo 2.0 to calculate viability. Error bars represent the standard deviation of four biological replicates. Treatments are relative to untreated cells. b. Human iPSC-derived cardiomyocytes were treated for 72 hours with vehicle or the indicated concentrations of JQ1 or ABBV-744, and the percentage of dead cells was quantified as the ratio of propidium iodide-positive cells (dead cells) over Hoechst-positive cells (all cells). Error bars represent the standard deviation of three biological replicates (six biological replicates for the vehicle condition). c, Primary human bronchial epithelial (NHBE) cells were treated with ABBV-744 at the indicated concentrations for 72 hours and toxicity was assessed using CellTiter-Glo 2.0. Error bars represent the standard deviation of four biological replicates. P-values determined using Mann-Whitney twotailed test. Treatments are relative to vehicle cells. 


\section{ARTICLES}

a

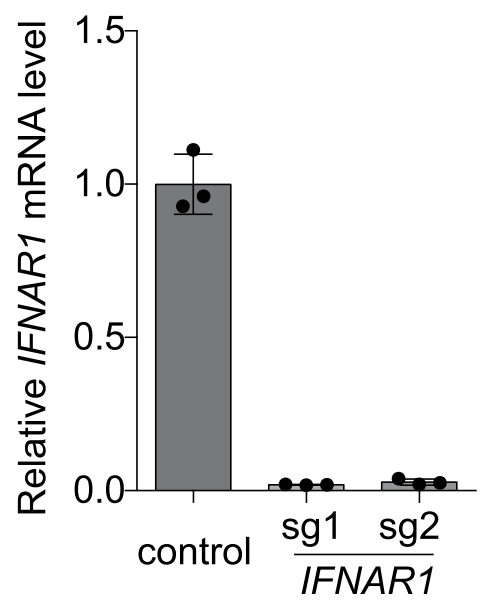

b

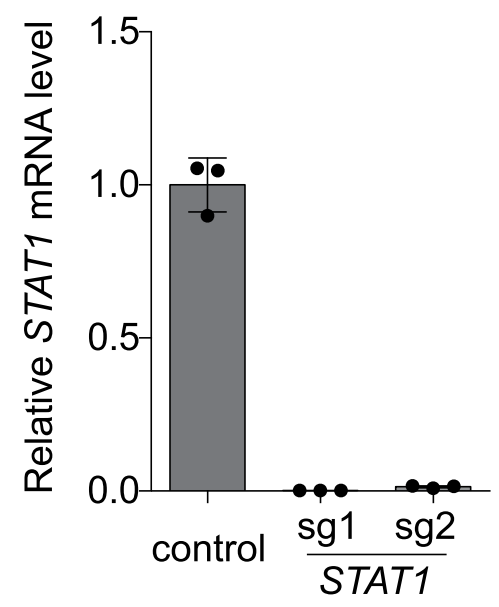

C

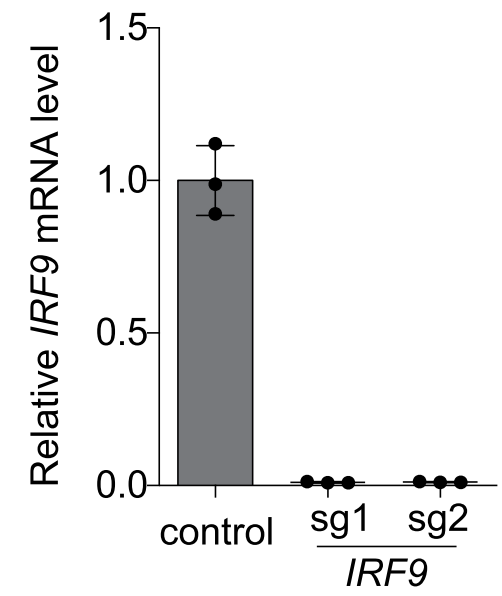

Extended Data Fig. 5 | Validation of knockdown of interferon regulators by CRISPRi. a-c, Calu-3 cells expressing sgRNAs knocking down genes essential for interferon signal transduction assayed for transcript levels of sgRNA targets relative to ACTB by qPCR. mRNA levels are fraction of control sgRNA. Error is the standard deviation of three biological replicates. 


\section{a}

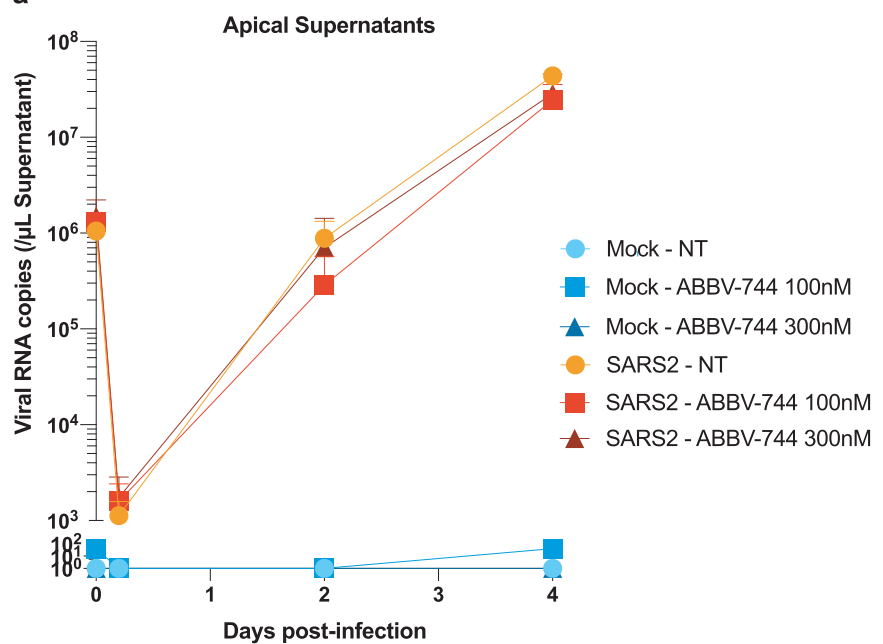

b

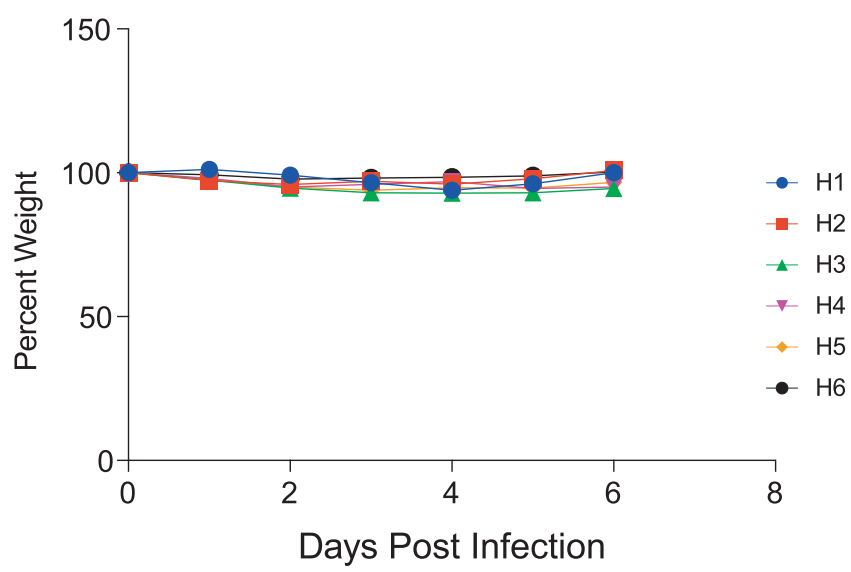

Extended Data Fig. 6 | Viral replication in apical supernatants of reconstructed human nasal epithelia cultures and bodyweight of hamsters throughout the course of SARS-CoV-2 infection. a-c, Calu-3 cells expressing sgRNAs knocking down genes essential for interferon signal transduction assayed for transcript levels of sgRNA targets relative to ACTB by qPCR. mRNA levels are fraction of control sgRNA. Error is the standard deviation of three biological replicates. A, Apical supernatants of either infected or mock-infected nasal epithelia treated with ABBV-744 at the indicated concentrations or not treated (NT) were isolated and assayed for SARS-CoV-2 N RNA content. Average of four biological quadruplicates are shown with error bars representing the standard deviation. b. Hamsters were weighed over the course of SARS-CoV-2 infection and weights were plotted as a percent of bodyweight on the day of infection. Inset, zoom in on body weight percent between 85 and 110 percent. 


\section{Reporting Summary}

Nature Research wishes to improve the reproducibility of the work that we publish. This form provides structure for consistency and transparency in reporting. For further information on Nature Research policies, see our Editorial Policies and the Editorial Policy Checklist.

\section{Statistics}

For all statistical analyses, confirm that the following items are present in the figure legend, table legend, main text, or Methods section.

$\mathrm{n} / \mathrm{a}$ Confirmed

$\bigotimes$ The exact sample size $(n)$ for each experimental group/condition, given as a discrete number and unit of measurement

$\bigotimes$ A statement on whether measurements were taken from distinct samples or whether the same sample was measured repeatedly

The statistical test(s) used AND whether they are one- or two-sided

Only common tests should be described solely by name; describe more complex techniques in the Methods section.

Х $\square$ A description of all covariates tested

Х $\square$ A description of any assumptions or corrections, such as tests of normality and adjustment for multiple comparisons

A full description of the statistical parameters including central tendency (e.g. means) or other basic estimates (e.g. regression coefficient)

AND variation (e.g. standard deviation) or associated estimates of uncertainty (e.g. confidence intervals)

For null hypothesis testing, the test statistic (e.g. $F, t, r$ ) with confidence intervals, effect sizes, degrees of freedom and $P$ value noted Give $P$ values as exact values whenever suitable.

Х $\square$ For Bayesian analysis, information on the choice of priors and Markov chain Monte Carlo settings

Х $\square$ For hierarchical and complex designs, identification of the appropriate level for tests and full reporting of outcomes

$\triangle \square$ Estimates of effect sizes (e.g. Cohen's $d$, Pearson's $r$ ), indicating how they were calculated

\section{Our web collection on statistics for biologists contains articles on many of the points above.}

\section{Software and code}

Policy information about availability of computer code

Data collection BD FACSDiva (version 8.01.1) was used to collect flow cytometry data and perform FACS

Data analysis Prism 6 was used for all plotting and data analysis other than the RNA-seq, CRISPRi screen and CUT\&RUN data analysis.

Flow data was analyzed in FlowJo(v10.4.0).

CUT\&RUN analysis was performed as previously described. Briefly, paired-end reads were mapped to the human genome GRCh38 using Bowtie2 (v2.3.4.1) with options: --end-to-end --very-sensitive --no-unal --no-mixed --no-discordant --phred33 -I 10 -X 1000. Sparse Enrichment Analysis for CUT\&RUN (SEACR, v1.3, https://seacr.fredhutch.org/) was used for peak calling. H3K4me3 and BRD2 peaks were normalized to IgG control. Published BRD2 ChIP-seq data in human lung cells was obtained from ChIP-Atlas (https://chip-atlas.org/). The Integrative Genomics Viewer (IGV, v2.9.4, igv.org) was used for visualization.

Raw sequencing reads from QuantSeq were trimmed using Trimmomatic43 (v0.39) and mapped to the human reference transcriptome (GRCh38, GENCODE Release 36) using Salmon44 (v1.3.0) to obtain transcript abundance counts. Gene-level count estimates were obtained using tximport45 (v1.18.0) with default settings. Subsequently, differential gene-expression analyses were performed using the gImQLFTest method implemented in the edgeR package46(v3.28.1). Cluster47 (v3.0) was used for hierarchical clustering and Java TreeView48 (v1.1.6r4) for visualization.

CRISPRi screen data analysis was performed with custom scripts as described in Tian, Ruilin et al. "CRISPR Interference-Based Platform for Multimodal Genetic Screens in Human iPSC-Derived Neurons." Neuron vol. 104,2 (2019): 239-255.e12. doi:10.1016/j.neuron.2019.07.014 using customs scripts, which are freely available at: https://kampmannlab.ucsf.edu/mageck-inc.

The ICE software from Synthego (v2, https://ice.synthego.com/\#/) was used to determine CRISPR knockout efficiency.

Binding and Expression Target Analysis (BETA, v1.0.7, http://cistrome.org/BETA/) was performed to identify direct BRD2 targets that were differentially expressed upon BRD2 knockdown. 
Policy information about availability of data

All manuscripts must include a data availability statement. This statement should provide the following information, where applicable:

- Accession codes, unique identifiers, or web links for publicly available datasets

- A list of figures that have associated raw data

- A description of any restrictions on data availability

Sequencing data are provided available on NCBI Gene Expression Omnibus (GEO) with the following accession numbers: GSE165025 (RNA sequencing data associated with Fig. 4), GSE182993 (CUT\&RUN data associated with Fig. 5), and GSE182994 (RNA sequencing data associated with Fig. 6f-h. Previously published BRD2 ChIP-seq data that were re-analysed here are available under accession code GSE113714 and GSE104481. Source data are provided with this study. All other data supporting the findings of this study are available from the corresponding author on reasonable request.

\section{Field-specific reporting}

Please select the one below that is the best fit for your research. If you are not sure, read the appropriate sections before making your selection.

\ Life sciences

Behavioural \& social sciences

Ecological, evolutionary \& environmental sciences

For a reference copy of the document with all sections, see nature.com/documents/nr-reporting-summary-flat.pdf

\section{Life sciences study design}

All studies must disclose on these points even when the disclosure is negative.

Sample size No sample size calculation was performed. As per standard practice in molecular and cell biology, we generally chose to do at least three replicates for each experiment.

Data exclusions No data was excluded from analysis

Replication $\quad$ All attempts at replication were successful. All experimental details necessary to replicate the results in other labs are provided in the Methods section. Experiments were replicated in at least technical triplicate. Most experiments were performed in at least biological triplicate.

Randomization Randomization does not apply to cell-based experiments, in which large numbers of cells from a given source were partitioned among experimental conditions. Hamsters were randomly assigned to treatment and control groups.

Blinding This is not relevant to our study, since no subjective rating of data was involved. All results involved equipment-based quantitative measurements rather than human-based evaluation or classification.

\section{Reporting for specific materials, systems and methods}

We require information from authors about some types of materials, experimental systems and methods used in many studies. Here, indicate whether each material, system or method listed is relevant to your study. If you are not sure if a list item applies to your research, read the appropriate section before selecting a response.

\begin{tabular}{l|l} 
Materials \& experimental syste \\
\hline$n / a$ & Involved in the study \\
\hline & $\bigotimes$ Antibodies \\
$\square$ & $\square$ Eukaryotic cell lines \\
$\square$ & $\square$ Animals and other organisms \\
$\square$ & $\square$ Clinical data \\
\hline
\end{tabular}

\begin{tabular}{l|l}
\multicolumn{2}{l}{ Methods } \\
\hline n/a & Involved in the study \\
$\square$ & $\square$ ChIP-seq \\
$\square$ & $\bigotimes$ Flow cytometry \\
$\square$ & $\square$ MRI-based neuroimaging
\end{tabular}

Antibodies

Antibodies used

Mouse monoclonal GAPDH (6C5) [Santa Cruz Biotech sc-32233]; Goat polyclonal ACE2 [R\&D Tech AF933]; Rabbit monoclonal BRD2 (EPR7642) [abcam 139690]; Donkey Anti-Goat-800 [LICOR 926-32214]; LICOR Donkey Anti-Mouse-680 [LICOR 926-68072]; HRP Donkey anti-rabbit [CST 7074P2] multiple lots of each antibody were used.

Validation 
Western blots of those same lines, when compared to WT, showed the absence of a band at the ACE2 molecular weight. 25 citations validation by manufacturer: correct size

Rabbit monoclonal BRD2 [abcam 139690]:

validation by manufacturer: knockout validated, https://www.abcam.com/hrp-brd2-antibody-epr7642-ab198536.html?

productWallTab=ShowAll

Mouse GAPDH

validation by manufacturer: 3095 citations, correct size. https://www.scbt.com/p/gapdh-antibody-6c5

\section{Eukaryotic cell lines}

Policy information about cell lines

Cell line source(s)

Calu3 cells were obtained from the UCSF Cell and Genome Core.

A vial of STR authenticated Caco-2 cells was obtained from the UCSF Cell and Genome Engineering Core (CGEC)

iPSCs (Allen Institute Cell Catalog: AICS90)

HEK293T (ATCC: CRL-3216)

NHEBs (MAttek NHBE-CRY)

A vial of A549 cells (ATCC: CCL-185) was obtained from Davide Ruggero's lab as a gift.

Authentication

Mycoplasma contamination

Commonly misidentified lines

(See ICLAC register)

\section{Cells were not authenticated for this study}

All the cell lines used in this study are not contaminated with mycoplasma. These cell lines were subject to mycoplasma detection once every 3 months using the UNiversial Mycoplasma Detection Kit (ATCC 30-1012KTM)

No commonly misidentified lines were used

\section{Animals and other organisms}

Policy information about studies involving animals; ARRIVE guidelines recommended for reporting animal research
Laboratory animals
Mesocricetus auratus, syrian golden hamster, male, 6-8 weeks old
Wild animals
The study did not involve wild animals
Field-collected samples
The study did not involve samples collected from the field
Ethics oversight
All experimental procedures and protocols were approved by the Institutional Animal Care and Use Committee at Icahn School of Medicine at Mount Sinai under protocol number IACUC\#20-0743

Note that full information on the approval of the study protocol must also be provided in the manuscript.

\section{Flow Cytometry}

Plots

Confirm that:

\The axis labels state the marker and fluorochrome used (e.g. CD4-FITC).

$\bigotimes$ The axis scales are clearly visible. Include numbers along axes only for bottom left plot of group (a 'group' is an analysis of identical markers).

\All plots are contour plots with outliers or pseudocolor plots.

$\bigotimes$ A numerical value for number of cells or percentage (with statistics) is provided.

\section{Methodology}

Sample preparation

Instrument

Software

Cell population abundance

Gating strategy

$\bigotimes$ Tick this box to confirm that a figure exemplifying the gating strategy is provided in the Supplementary Information.
Calu3 cells were trypsinized with TrypLE and resuspended in RPMI1640 for flow cytometry

\section{BD LFSRFortessa X14}

BD FACSdive (Version 8.0.1.1) and FlowJo (Version 10.0.7)

Cell populations are differentiated by fluorescent markers. All populations have sufficient events.

Cells were gated based on FSCA/SSCA to differentiate live from dead cells. This population was then gated based on FSC-W $x$ FSC-A to gate single cells. Gating strategy is shown in supplemental figure 2. 